University of Massachusetts Amherst

ScholarWorks@UMass Amherst

2006

\title{
Chandra ACIS Spectroscopy of N157B: A Young Composite Supernova Remnant in a Superbubble
}

Y Chen

QD Wang

University of Massachusetts - Amherst

EV Gotthelf

B Jiang

Y-H Chu

See next page for additional authors

Follow this and additional works at: https://scholarworks.umass.edu/astro_faculty_pubs

Part of the Astrophysics and Astronomy Commons

\section{Recommended Citation}

Chen, Y; Wang, QD; Gotthelf, EV; Jiang, B; Chu, Y-H; and Gruendl, R, "Chandra ACIS Spectroscopy of N157B: A Young Composite Supernova Remnant in a Superbubble" (2006). Astronomy Department Faculty Publication Series. 1049.

$10.1086 / 507017$ 
Authors

Y Chen, QD Wang, EV Gotthelf, B Jiang, Y-H Chu, and R Gruendl 
To Appear in the ApJ 10 OCtober 2006, v650N1 issue

Preprint typeset using $\mathrm{LATE}_{\mathrm{E}} \mathrm{X}$ style emulateapj v. 6/22/04

\title{
CHANDRA ACIS SPECTROSCOPY OF N157B - A YOUNG COMPOSITE SUPERNOVA REMNANT IN A SUPERBUBBLE
}

\author{
Yang Chen $^{1}$, Q. Daniel Wang ${ }^{2,3}$, E. V. Gotthelf ${ }^{4}$, Bing Jiang ${ }^{1}$, You-Hua Chu ${ }^{5}$, And Robert Gruendl ${ }^{5}$ \\ To appear in the ApJ 10 October 2006, v650n1 issue
}

\begin{abstract}
We present a Chandra ACIS observations of N157B, a young supernova remnant (SNR) located in the 30 Doradus star-formation region of the Large Magellanic Cloud. This remnant contains the most energetic pulsar known (J053747.39-691020.2; $\dot{E}=4.8 \times 10^{38} \mathrm{ergs} \mathrm{s}^{-1}$ ), which is surrounded by a $\mathrm{X}$-ray bright nonthermal nebula that likely represents a toroidal pulsar wind terminal shock observed edge-on. Two of the eight point-like X-ray sources detected in the observation are shown to have near-IR and optical counterparts (within 0.5 offsets), which are identified as massive stellar systems in the Cloud. We confirm the non-thermal nature of the comet-shaped X-ray emission feature and show that the spectral steepening of this feature away from the pulsar is quantitatively consistent with synchrotron cooling of shocked pulsar wind particles flowing downstream at a bulk velocity close to the speed of light. Around the cometary nebula we unambiguously detect a spatially-resolved thermal component, which accounts for about $1 / 3$ of the total $0.5-10 \mathrm{keV}$ flux from the remnant. This thermal component is distributed among various clumps of metal-enriched plasma embedded in the low surface brightness X-ray-emitting diffuse gas. The relative metal enrichment pattern suggests that the mass of the supernova progenitor is $\gtrsim 20 M_{\odot}$. A comparison of the X-ray data with Hubble Space Telescope optical images now suggests that the explosion site is close to a dense cloud, against which a reflection shock is launched. The interaction between the reflected material and the nebula has likely produced both its cometary shape and the surrounding thermal emission enhancement. SNR N157B is apparently expanding into the hot low-density interior of the surrounding superbubble formed by the young OB association LH99, as revealed by Spitzer mid-infrared images. This scenario naturally explains the exceptionally large sizes of both the thermal and nonthermal components as well as the lack of an outer shell of the SNR. However, the real situation in the region is likely to be more complicated. We find that a partially-round-shaped soft X-ray-emitting clump with distinct spectral properties may result from a separate oxygen-rich remnant. These results provide a rare glimpse into the SNR structure and evolution in a region of recent star-formation.
\end{abstract}

Subject headings: pulsars: general — pulsars: individual (PSR J0537-6910) - X-rays: general supernova remnant: individual (N157B, SNR 0538-691) — galaxies: individual (Large Magellanic Cloud)

\section{INTRODUCTION}

The evolution of a supernova remnant (SNR) depends sensitively on its environment. Most massive stars are expected to be born in $\mathrm{OB}$ associations and end their lives in an environment altered by strong ionizing radiation and mechanical energy feedback from fast stellar winds and supernovae (SNe). This feedback produces superbubbles filled with low-density hot gas. An SNR inside such a superbubble will be difficult to observe (e.g., Tang \& Wang 2005). A classical example is the Crab SNR, which shows little detectable emission from its blastwave-heated gas and is believed to be expanding in a low-density medium. Without the synchrotron radiation from its pulsar wind nebula (PWN), the Crab SNR would not have been identified. Similar SNRs dominated

1 Department of Astronomy, Nanjing University, Nanjing 210093, P.R.China

2 Department of Astronomy, B619E-LGRT, University of Massachusetts, Amherst, MA 01003

${ }^{3}$ Institute for Advanced Study, Einstein Drive, Princeton, NJ 08540

${ }_{4}$ Columbia Astrophysics Laboratory, Columbia University, 550 West 120 St, New York, NY 10027

5 Astronomy Department, University of Illinois at UrbanaChampaign, 1002 West Green Street, Urbana, IL 61801 by radiation from PWNe are called "Crab-like". Physically, Crab-like SNRs are not very different from the "composite" remnants, which show comparable thermal and nonthermal components or show a shell-like structure with a centrally-filled morphology. The increasing sensitivity of X-ray observations has made it possible to detect more shell-like and thermal features around Crablike SNRs. For example, the Crab-like SNR G21.5-0.9 has recently been shown to be surrounded by ejecta- or blastwave-dominated features (Slane et al. 2000; Matheson \& Safi-Harb 2005). In this paper, we present evidence that the Crab-like SNR N157B, also known as SNR 0538691 , exhibits thermal X-ray emission as well and is thus a composite SNR.

SNR N157B is particularly interesting, because of its co-existence with a young OB association LH99 in the Large Magellanic Cloud (LMC) (Lucke \& Hodge 1970; Chu et al. 1992). The SNR has long been classified to be Crab-like and contains the fastest known young pulsar PSR J0537-6910 with a period of $16 \mathrm{~ms}$ (Marshall et al. 1998). The SNR appears to have an exceptionally large physical size in both radio and X-ray $(\sim 20-30 \mathrm{pc}$; Lazendic et al. 2000; Wang \& Gotthelf 1998, WG98 hereafter), although the outer boundaries have not yet been well determined. Of particular interest is how the struc- 
ture and evolution of SNR N157B is affected by the presence of the OB association LH99. Is the SNR expanding in a low-density hot medium, as may be expected in a superbubble created by the OB association? Addressing such questions is important in understanding both the fate of the feedback from massive stars and the physics involved in the evolution of such an SNR (e.g., Tang \& Wang 2005).

Previous X-ray studies based on data with low angular resolutions were concentrated on the overall spectral and morphological characterization of N157B. Using $A S C A$ and ROSAT data, WG98 found that the X-ray emission arises from a bright cometary-shaped nebula, headed by a compact source embedded in a bright bar of nonthermal emission, with a long tail of diffuse emission trailing on one side aligned perpendicular to the bar. This morphology is reminiscent of those associated with trailing plerions in SNRs G327.1-1.1 (Sun, Wang, \& Chen 1999), W44 (Frail et al. 1996), and IC443 (Olbert et al. 2001; Gaensler et al. 2006), as well as the PWN around PSR B1757-24 near SNR G5.4-1.2 (the Duck X-ray Nebula; Kaspi et al. 2001). Using a Chandra HRC observation with high angular and timing resolutions, Wang et al. (2001) were able to pinpoint (to arcsec precision) the location of PSR J0537-6910, which is still undetected in any other wavelength band (e.g. Mignani et al. 2005; Crawford et al. 2005). The nebula was shown to consist of primarily a $\sim 0.6 \mathrm{pc} \times 1.7 \mathrm{pc}$ compact bar-like feature around the pulsar and a tail $\gtrsim 5 \mathrm{pc}$ long. This morphology indicates that the bar represents the reverse shock of a toroidal wind from PSR J0537-6910 and that the ambient pressure confinement of the nebula is largely one-sided.

The one-sided confinement of the N157B nebula is thought to be a result of the pulsar motion relative to the ambient medium. This hypothesis seems to be consistent with the displacement of the pulsar from an extended radio continuum emission peak, mostly representing the accumulation of pulsar wind particles, assumed to be mainly electrons and positrons (WG98). Based on 2-D hydrodynamic simulations, van der Swaluw, Downes, \& Keegan (2004) and van der Swaluw (2004) have concluded that a typical PWN evolves through three major stages: 1) expanding supersonically in freely expanding SN ejecta; 2) interacting with the reverse shock of the SNR, oscillating and relaxing to a subsonic expansion in the reverse-shock heated ejecta; and 3) heading into the general ISM, supersonically. In both the first and the third stages, one may expect a bow shock because of supersonic motion. They suggest that the PWN of the N157B nebula is currently in the second stage; the tail of the nebula is a pulsar wind bubble that was elongated owing to the pulsar motion and was later crushed by the reverse shock of the SNR. The reverse shock heats a shell of ejecta swept-up by the expansion of the PWN and bounces back into the already heated remnant materials, forming a parabolic layer (see Fig. 2 in van der Swaluw 2004). In this scenario, the relative motion between the pulsar and its ambient medium does not need to be supersonic. Of course, the reality could be much more complicated than what is assumed in the simulations. The region shows clearly large density and temperature inhomogeneities that could have significantly affected the dynamics of both the SNR and the PWN (Fig. 1, b). In short, the exact nature of the confinement as well as the physical properties of the nebula are still very uncertain.

Existing studies have also shown evidence for a weak thermal emission component of N157B. Much of the evidence comes from the spectral decomposition of the overall emission from the SNR. The thermal emission arises over a region more extended than the PWN, but only very limited spatial analysis has been presented previously (WG98; Dennerl et al. 2001; Townsley et al. 2006). It is thus of great interest to examine the physical, chemical, and morphological properties of the thermal emission and to determine how they are related to the PWN and to the $\mathrm{OB}$ association and superbubble environment.

To obtain a fresh look of the above issues, we have acquired an on-axis Chandra ACIS-S observation of N157B (Fig. 2), allowing for a detailed spatially-resolved X-ray spectroscopy of the SNR. We have also analyzed the Xray data together with complementary archival images from recent Hubble Space Telescope (HST) Advanced Camera for Surveys (ACS) and Spitzer Space Telescope Infrared Array Camera (IRAC) observations. In $\S 2$, we briefly describe the observation and data calibration, and present our analysis and results. The effects of the superbubble and density inhomogeneity, the origin of the thermal emission, and the nature of various nonthermal features of the PWN are discussed in $\S 3$. We summarize our results and conclusions in $\S 4$. Throughout this paper, we adopt the distance of N157B as $50 \mathrm{kpc}$ (thus $1^{\prime \prime}$ corresponds to $0.25 \mathrm{pc}$ ). Statistical errors are all presented in the $90 \%$ confidence level.

\section{OBSERVATIONS AND DATA ANALYSIS}

The X-ray study reported here is based on our Chandra observation of N157B (ObsID 2783). The data were collected in two exposures, obtained on 2002 August 2324 and on 2002 October $28-30$, for a combined duration of $53 \mathrm{ks}$. The data from the two exposures were merged through the pipeline processing and no additional alignment is attempted. Because of the separation in time, the relative roll angle of these exposures is $65 \mathrm{deg}$ (Fig. 2). Data were collected with ACIS-S S3 in the focal plane using the 1/4-chip sub-array mode (with a frame readout time of $0.8 \mathrm{~s}$ ) to minimize photon pile-up associated with the bright pulsar. The first part of the observation (30 ks exposure) has been used by Mignani et al. (2005) in a primarily optical study of PSR J0537-6910, including a search for its optical counterpart and an X-ray spectral analysis.

We reprocess the event files (from Level 1 to Level 2) for the observation using the updated CIAO data processing software. After removing flares with count rates greater than 1.2 times the mean light curve value, a net exposure of $48 \mathrm{ks}$ remains and is used for subsequent analysis. For the spectral analysis, individual spectra are adaptively binned to achieve a background-subtracted signal-to-noise ratio of 5 , unless otherwise noted. The XSPEC spectral fitting software is used throughout. For the foreground absorption, we use the cross-sections of Morrison \& McCammon (1983) and assume solar abundances.

Fig. 3 presents the overall Chandra X-ray intensity image in the soft $(0.3-1.5 \mathrm{keV})$ and hard $(1.5-7.0 \mathrm{keV})$ energy bands, which clearly demonstrate the energy dependence of the SNR morphology. The images do not 


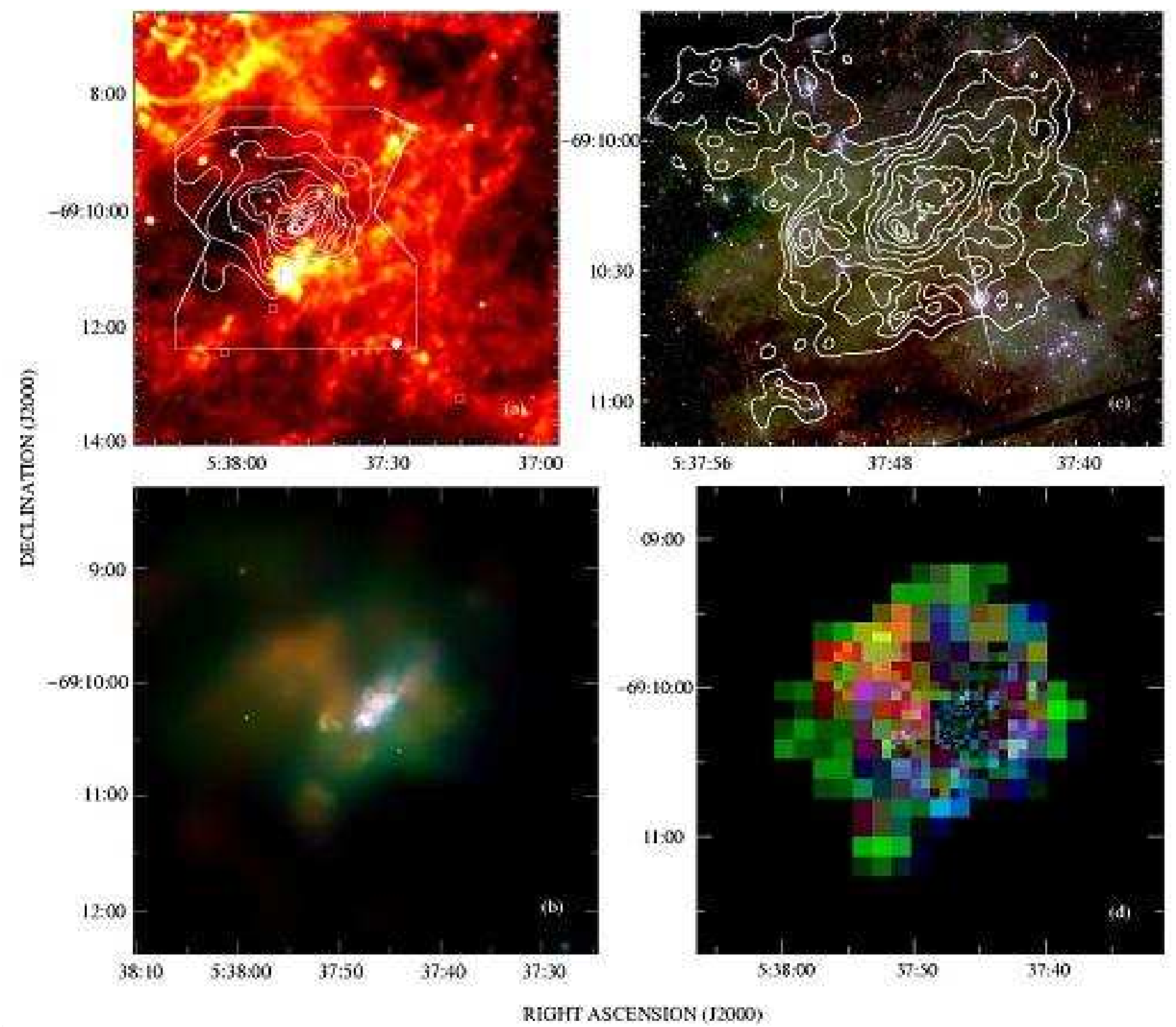

FIG. 1.- (a) A Spitzer global view of the N157B region in mid-infrared (IR) (8 $\mu \mathrm{m}$; Fazio et al. 2004) with overlaid Chandra ACIS-S intensity contours at $2,3,4,6,9,13,18,25,50,100,200,500$, and $1000 \times 10^{-2}$ counts s ${ }^{-1} \operatorname{arcmin}^{-2}$. The outer boundary of the contour map shows the region covered in (b). The small square boxes mark discrete X-ray sources detected. (b) Color-coded multi-band ACIS-S intensity images of N157B in the 0.3-0.7 keV (red), 0.7-1.5 keV (Green), and 1.5-7 keV (blue) bands. (c) ACIS-S intensity contours at 12, $20,30,50,100,250,800,2500,10000 \times 10^{-2}$ counts s${ }^{-1}$ arcmin $^{-2}$ overlaid on an HST ACS close-up of the N157B central region in optical: F814W (color-coded in red), F555W (green), and F435W (blue). (d) Equivalent width (EW) images of the emission lines: O (in red), Ne (green), and $\mathrm{Mg}$ (blue) in square-root brightness scales; the line energies are listed in Table 1 and the image for each band is adaptively rebinned to include at least 10 counts per pixel. The X-ray images in (a) and (b) are smoothed with the CIAO routine csmooth, whereas the image in (c) is smoothed with a Gaussian kernel adjusted adaptively to achieve a count-to-noise ratio of 6 .

show a typical rim-brightened outer SNR shell; therefore, the overall size of the diffuse X-ray emission is uncertain. Here we will adopt a diameter of the remnant as $\sim 100^{\prime \prime}$. The diffuse emission also exhibits various lumpy sub-structures. Intensity maps and corresponding exposure maps were also generated in four broad energy bands, $0.3-0.7 \mathrm{keV}, 0.7-1.5 \mathrm{keV}, 1.5-3.0 \mathrm{keV}$, and $3.0-7.0 \mathrm{keV}$ (Wang 2004). These maps are used to create the flat-fielded count rate images, which are typically smoothed with an adaptive filter (using the program csmooth implemented in the CIAO software package) to achieve a broad-band $(0.3-7 \mathrm{keV})$ signal-to-noise ratio of $\sim 3$.
To compare the distribution of the X-ray emission with its interstellar environment of N157B, we have used HST ACS/WFC and Spitzer IRAC images. The pipelineprocessed ACS images from HST program 9471 (PI: Mignani), taken with the F435W, F555W, and F814W filters, were retrieved from the MAST archive and are presented without further processing. Spitzer observed the 30 Dor region with the IRAC (Fazio et al. 2004) as part of programs 63, 1032, and 20203 (PIs: Houck, Brandl, and Meixner, respectively). Each program used the IRAC mapping mode to obtain maps of the region with multiple dithered $12 \mathrm{~s}$ exposures at each location in the map. The Basic Calibrated Data (BCDs) from these 


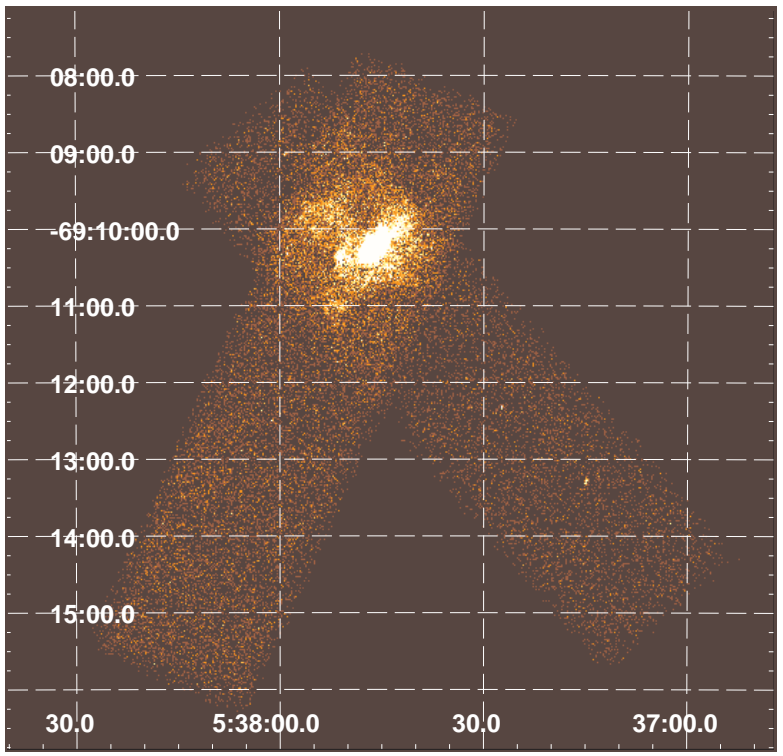

FIG. 2.- The raw data image of the Chandra ACIS-S observation of SNR N157B. The coordinate axes are for R.A. (J2000) and Dec. (J2000), respectively.

TABLE 1

ENERGY BOUNDS OF THE EW IMAGES

\begin{tabular}{lccc}
\hline \hline Elements & $\begin{array}{c}\text { Line } \\
(\mathrm{eV})\end{array}$ & $\begin{array}{c}\text { Low }^{\mathrm{a}} \\
(\mathrm{eV})\end{array}$ & $\begin{array}{c}\text { High }^{\mathrm{a}} \\
(\mathrm{eV})\end{array}$ \\
\hline $\mathrm{O}$ & $590-740$ & $300-550$ & $1160-1260$ \\
$\mathrm{Ne}$ & $870-1140$ & $750-870$ & $1160-1260$ \\
$\mathrm{Mg}$ & $1290-1430$ & $1160-1260$ & $1620-1720$ \\
\hline
\end{tabular}

a The low- and high-energy ranges around the selected line energies used to estimate the underlying continuum.

programs were combined using standard routines in the MOPEX software package to obtain images with $\sim 180 \mathrm{~s}$ integration time at all locations where the programs overlap. More information on the instruments, pipeline processing, and the MOPEX package can be found at the Spitzer Science Center's Observer Support website ${ }^{6}$.

Fig. 1. 1presents various perspectives of SNR N157B and its environment. The X-ray photon energy dependence is illustrated in tricolor images, for broad-band intensities and for equivalent widths (EWs) of key emission lines listed in Table 1. The comet-shaped nebula, as presented in WG98 and Wang et al. (2001), stands out as the brightest and hardest X-ray feature in the field. Surrounding the nebula is a large-scale diffuse emission with relatively low-surface brightness and soft spectral characteristics. These smoothed X-ray images are also compared with both optical and mid-IR maps to illustrate their complex relationship.

Point sources in the field were detected following the procedure detailed in Wang (2004) using a combination of algorithms: wavelet, sliding box, and maximum likelihood centroid fitting in the above energy bands. The

\footnotetext{
${ }^{6}$ http://ssc.spitzer.caltech.edu/ost.
}

source count rate is estimated based on the net counts within the $90 \%$ energy-encircled radius (EER) of the telescope/ACIS point-spread function (PSF; Jerius et al. 2000). Table 2 lists our detected point-like sources whose positions are marked in Fig. 1h. Because of the exposure correction for the exposure is very small in the subarry (smaller than the systematic errors involved in calculating the count rates), the actual count rates without correction are included in the table.

We search for possible counterparts in the SIMBAD, NED, and Two Micron All Sky Survey (2MASS) databases (Skrutskie et al. 2006) as well as in various optical images. We find two optical stellar counterparts, 128 (type O5 f*, V=14.26) and 1-98 [O5 V(f(f)), V=13.92; Schild \& Testor 1992], for the X-ray sources \# 3 and 7 , respectively (Table2). The most accurate ( 0 ". 1 RMS) positions of these two stars are obtained from 2MASS: R.A. (J2000), Dec. $(\mathrm{J} 2000)=5^{\mathrm{h}} 37^{\mathrm{m}} 37^{\mathrm{s}} .97,-69^{\circ} 10^{\prime} 14^{\prime \prime} \cdot 7$ for $1-$ 28 and $5^{\mathrm{h}} 37^{\mathrm{m}} 59.43,-69^{\circ} 9^{\prime} 1^{\prime \prime} \cdot 1$ for $1-98$; the offsets from the respective X-ray sources are $0 . \prime 1$ and $0{ }^{\prime \prime} 5$, consistent with their position uncertainties (Table 21). Therefore, the absolute astrometry accuracy of the X-ray observation is likely better than 0.5 . Except for the pulsar (\# 4 ), the other detected X-ray sources are all quite faint. Assuming the X-ray spectrum as a thermal plasma with a temperature of $0.6 \mathrm{keV}$ (for soft case) and $2 \mathrm{keV}$ (for hard case), respectively, typical for normal early-type stars, and a foreground absorption of $N_{\mathrm{H}} \sim 6 \times 10^{21} \mathrm{~cm}^{-2}$ in the region, we estimate the $0.3-8 \mathrm{keV}$ luminosities of the two X-ray sources as $\sim(1.2-3.6) \times 10^{33} \mathrm{ergs} \mathrm{s}^{-1}$ based on the count rates in Table 2 These luminosities are higher than $10^{33} \mathrm{ergs} \mathrm{s}^{-1}$, indicating that the X-ray sources most probably represent massive colliding stellar wind binaries, while they may still possibly be individual early O-type or WR stars (e.g., Oskinova 2005). The source \# 6 , very soft and without an optical counterpart, may be a foreground cataclysmic variable. Other sources are at larger off-pulsar distances and are probably also not related to N157B.

\subsection{Spectral Properties}

We extracted spectra for analysis from the various features marked in the raw count image shown in Fig. 目 This figure shows some details that have been smoothed out in the previous images. The overall spectrum of the SNR is taken from a large rectangular region (labeled "ENT" in Fig. 4), including the pulsar. The background spectrum is extracted from the southern and northern rectangular regions (B1 and B2) marked in Fig. 4 Because of the uncertainty of the actual extent of SNR, the background regions (B1 and B2) may still be contaminated by the diffuse emission from the remnant. The background-subtracted spectrum is shown in Fig. 5

Though nearly featureless above $\sim 2 \mathrm{keV}$, the spectrum shows evidence for the $\mathrm{K} \alpha$ emission lines of O VIII $(\sim 0.65 \mathrm{keV})$, Ne IX $(\sim 0.92 \mathrm{keV})$ and $\mathrm{Ne} \mathrm{X}(\sim 1.05 \mathrm{keV})$, Mg XI $(\sim 1.34 \mathrm{keV})$, and possibly Si XIII $(\sim 1.87 \mathrm{keV})$. We fit the spectrum with a power law plus a nonequilibrium ionization (NEI) thermal plasma that characterizes the parameters and ionization timescale $\left(n_{e} t_{i}\right)$ of the hot gas (Borkowski, Lyerly, \& Reynolds 2001). 

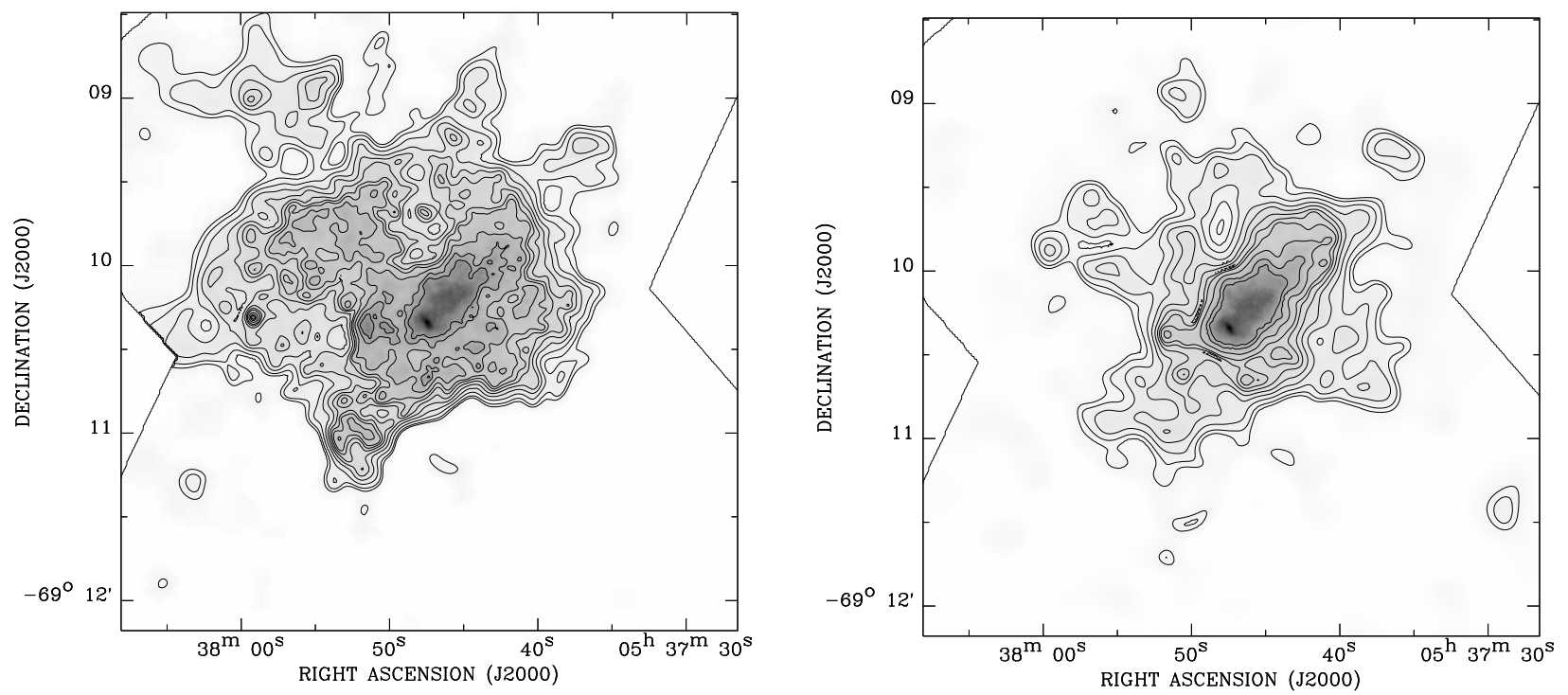

FIg. 3.- Chandra ACIS-S images of SNR N157B in the $0.3-1.5 \mathrm{keV}$ band (left panel) and the $1.5-7 \mathrm{keV}$ band. The images are smoothed with a Gaussian with its size adjusted adaptively to achieve a count-to-noise ratio of 6 . The contours are at $3,4,6,9,13,18$, $24,31,50,100$, and $200 \sigma$ above a background of $1.5 \times 10^{-2}$ counts s ${ }^{-1}$ arcmin $^{-2}$. The outer boundaries of the sub-array boundaries are outlined.

TABLE 2

Chandra SOURCE LisT

\begin{tabular}{|c|c|c|c|c|c|c|}
\hline $\begin{array}{l}\text { Source } \\
\text { (1) }\end{array}$ & $\begin{array}{c}\text { CXOU Name } \\
(2)\end{array}$ & $\begin{array}{c}\delta_{x}\left({ }^{\prime \prime}\right) \\
(3)\end{array}$ & $\begin{array}{c}\mathrm{CR}\left(\operatorname{cts~ks}^{-1}\right) \\
(4)\end{array}$ & $\begin{array}{c}\mathrm{HR} \\
(5)\end{array}$ & $\begin{array}{c}\mathrm{HR} 1 \\
(6)\end{array}$ & $\begin{array}{c}\text { Flag } \\
(7)\end{array}$ \\
\hline 1 & J053715.18-691316.6 & 0.7 & $2.49 \pm 0.40$ & $0.67 \pm 0.13$ & - & B \\
\hline 2 & J053727.56-691218.4 & 0.6 & $1.22 \pm 0.27$ & - & - & B \\
\hline 3 & J053737.97-691014.8 & 0.4 & $0.32 \pm 0.10$ & - & - & $\mathrm{B}$ \\
\hline 4 & J053747.39-691020.2 & 0.2 & $205.83 \pm 2.30$ & $0.36 \pm 0.01$ & $0.92 \pm 0.01$ & $\mathrm{H}$ \\
\hline 5 & J053751.79-691143.3 & 0.7 & $0.30 \pm 0.11$ & - & - & $\mathrm{H}$ \\
\hline 6 & J053759.15-691018.4 & 0.3 & $0.83 \pm 0.15$ & $-0.89 \pm 0.13$ & $0.97 \pm 0.06$ & $\mathrm{~B}$ \\
\hline 7 & J053759.48-690901.6 & 0.4 & $0.62 \pm 0.20$ & - & - & $\mathrm{B}$ \\
\hline 8 & J053801.42-691229.1 & 0.6 & $0.74 \pm 0.18$ & - & - & B \\
\hline
\end{tabular}

Note. - The definition of the bands: 0.3-0.7 (S1), 0.7-1.5 (S2), 1.5-3 (H1), and 3-7 keV (H2). In addition, $\mathrm{S}=\mathrm{S} 1+\mathrm{S} 2, \mathrm{H}=\mathrm{H} 1+\mathrm{H} 2$, and $\mathrm{B}=\mathrm{S}+\mathrm{H}$. Column (1): Generic source number. (2): Chandra X-ray Observatory (unregistered) source name, following the Chandra naming convention and the IAU Recommendation for Nomenclature (e.g., http://cdsweb.u-strasbg.fr/iau-spec.html). (3): Position uncertainty, including an $1 \sigma$ statistical error calculated from the maximum likelihood centroiding and an approximate off-axis angle $(r)$ dependent systematic error $0^{\prime \prime} 2+1^{\prime \prime} 4\left(r / 8^{\prime}\right)^{2}$ (an approximation to Fig. 4 of Feigelson et al. 2002), which are added in quadrature. (4): On-axis source broad-band count rate - the sum of the exposure-corrected count rates in the four bands. (5-6): The hardness ratios defined as $\mathrm{HR}=(\mathrm{H}-\mathrm{S} 2) /(\mathrm{H}+\mathrm{S} 2)$, and $\mathrm{HR} 1=(\mathrm{S} 2-\mathrm{S} 1) / \mathrm{S}$, listed only for values with uncertainties less than 0.2. (7): The labels "B", "S", or "H" mark the bands in which a source is detected with the most accurate position that is adopted in Column (2).

Our fit allows the abundances of the above significant line-emitting elements to vary, while other metal elements are set to be 0.3 solar, typical for the LMC (Russel \& Dopita 1992).

The fit results are summarized in Table 3 The luminosity of the nonthermal $0.5-10 \mathrm{keV}$ emission, including the contributions from PSR J0537-6910 and the PWN, is $L_{\mathrm{nt}} \sim 4.4 \times 10^{36} \mathrm{ergs} \mathrm{s}^{-1}$, twice that of the thermal emission $L_{\mathrm{th}} \sim 2.2 \times 10^{36} \mathrm{ergs} \mathrm{s}^{-1}$. Clearly, the overall spectrum of N157B is dominated by the power-law emission, particularly at high energies $(\gtrsim 2 \mathrm{keV})$. As a comparison, the overall luminosity of N157B is independently estimated as $4.2 \times 10^{36} \mathrm{ergs} \mathrm{s}^{-1}(0.5-8 \mathrm{keV})$ from the Chandra ACIS-I observation applying a collisional ion- ization model to the thermal component (Townsley et al. 2006). The thermal component is found to be neon-rich and is also slightly over-abundant in oxygen and magnesium, in respect to LMC values, indicating that much of the thermal emission arises from the SN ejecta.

To see where the metal-rich materials are located, we present the EW images of the $\mathrm{O}, \mathrm{Ne}$, and $\mathrm{Mg}$ lines in Fig. 11. These images are constructed in a method similar to those used by Hwang et al. (2000) and Park et al. (2002). A major difference of our method from theirs is that we rebin the data using an adaptive mesh with each bin including at least 10 counts(see Warren et al. 


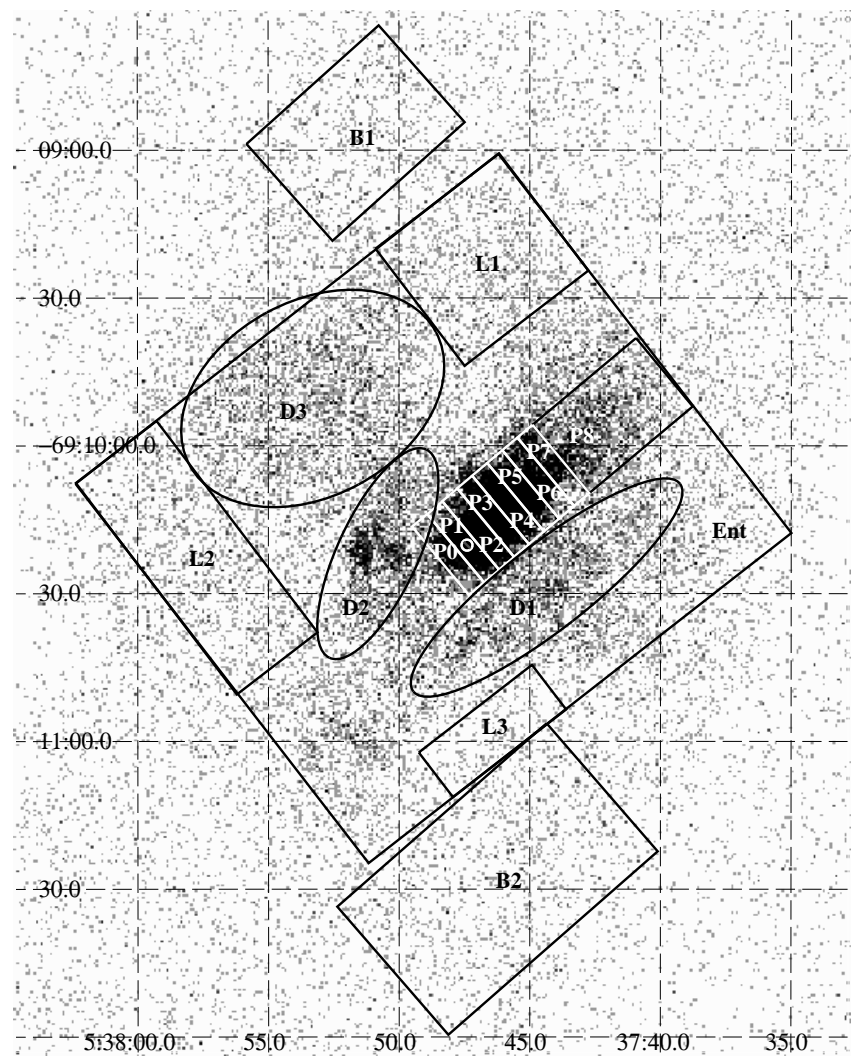

Fig. 4.- Raw ACIS-S image of N157B. The labeled regions are used for spectrum extraction. The small circle in Region P1 encircles the emission of PSR J0537-6910, and other point sources are removed. The coordinate axes are for R.A. (J2000) and Dec. (J2000), respectively.

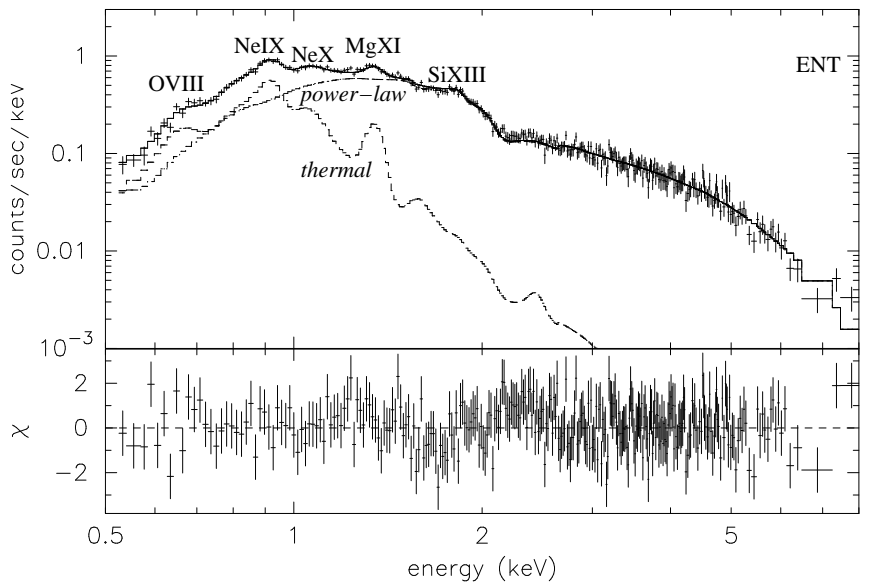

FIG. 5.- The overall spectrum of SNR N157B fitted with the model of a power-law plus an NEI thermal plasma. The lower panel (likewise also in other spectral plots) shows the residuals of the data minus the model in units of $\sigma$. The two spectral components are also shown separately.

2003 for similar binning). The figure shows that the line emission is distributed over a broad region, except for the cometary X-ray nebula, which is dominated by nonthermal emission and appears featureless. The Ne emission (in green) has the largest extent, while the $\mathrm{Mg}$ line (in blue) is strong mostly near the southwestern edge of the nebula (Region D1), while the O line (in red) is concen-
TABLE 3

Spectral Fit for the Entire SNR

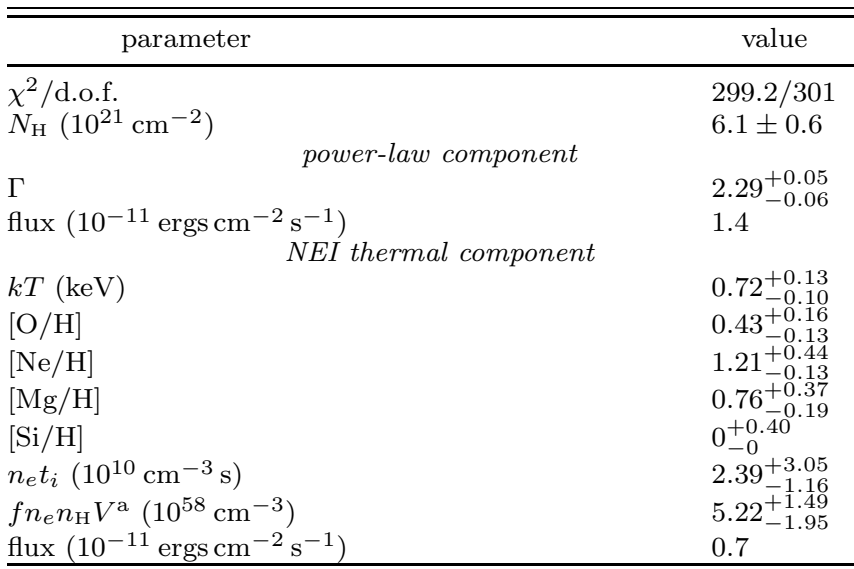

Note. - The unabsorbed fluxes are in the $0.5-10 \mathrm{keV}$ band. The net count rate is $1.090 \pm 0.005$ counts $^{-1}$.

a Quantity $f n_{e} n_{\mathrm{H}} V$ is the volume emission measure of the hot gas, where $f$ denotes the filling factor.

trated in a bright northeast patch (D3).

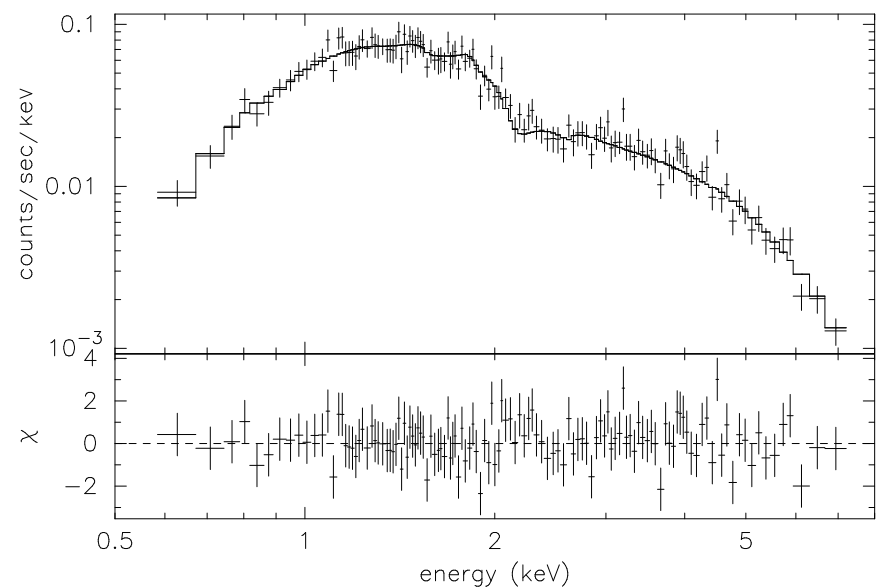

FIG. 6.- ACIS-S spectrum of PSR J0537-6910 and the best-fit power-law.

\subsection{PSR J0537-6910}

The location of PSR J0537-6910 corresponds to the emission peak of the N157B nebula. The position listed in Table 2 is consistent with the time-resolved peak of the pulsed emission from the pulsar $\left(5^{\mathrm{h}} 37^{\mathrm{m}} 47^{\mathrm{s}} .36\right.$, $69^{\circ} 10^{\prime} 20^{\prime \prime} .4$; Wang et al. 2001; see also Mignani et al. 2005). We extract a spectrum of PSR J0537-6910 or the core of the nebula bar from a circle of $1^{\prime \prime}$ radius $(90 \%$ EER) centered on the emission peak and a background from the bar minus the circle (Region P1 in Fig. (4). We fit the spectrum with a power-law model (Fig. [6 no evidence is found for any significant pile-up, based on the model given by Davis 2001). The fit is satisfactory $\left(\chi^{2} /\right.$ d.o.f. $\left.=110.0 / 125\right)$ and gives the best-fit power law index as $\Gamma=1.73_{-0.06}^{+0.11}$ and the foreground absorption column density as $N_{\mathrm{H}}=5.6_{-0.3}^{+0.5} \times 10^{21} \mathrm{~cm}^{-2}$. The 
absorption-corrected flux is $\sim 1.9 \times 10^{-12} \mathrm{ergs} \mathrm{cm}^{-2} \mathrm{~s}^{-1}$ in the $0.5-10 \mathrm{keV}$ band (or a luminosity of $\sim 5.7 \times$ $\left.10^{35} \mathrm{ergs} \mathrm{s}^{-1}\right)$. The results are consistent with the preliminary results given by Mignani et al. (2005). The power law index is also consistent with that measured for the pulsed X-ray emission from the pulsar $\left(\sim 1.6_{-0.3}^{+0.4}\right)$, based on the ASCA and RXTE spectra (Marshall et al. 1998). Assuming the same power law $(\Gamma=1.73)$, we find that the pulsed fraction is $47 \%$ in the $0.5-10 \mathrm{keV}$ band, compared to $40 \%$ in the $0.2-4 \mathrm{keV}$ band (Wang et al. 2001).

In the Spitzer IRAC images there are no point sources at the location of the pulsar. The upper limits for the detection of a point source with $3 \sigma$ confidence are flux densities of $22,28,53$, and $143 \mu \mathrm{Jy}$ in the IRAC $3.6,4.5$, 5.8 , and $8.0 \mu \mathrm{m}$ bands, respectively. Similarly, no radio or optical counterpart has been detected for the pulsar (Crawford et al. 2005; Mignani et al. 2005).

\subsection{Diffuse Emission: Nonthermal Component}

Our analysis of the global diffuse X-ray distribution shows that the cometary nebula has a hard and nearly featureless spectral characteristics, confirming the conjecture made in the previous studies (e.g., WG98). Fig. [7 further shows some interesting details that have not clearly been identified before. These include evidence of a halo around the bar that is most apparent in front of the bar and extends about $5^{\prime \prime}$ from the pulsar. In the $0.3-1.5 \mathrm{keV}$ band, the new data also show considerable substructures in the tail. However, the counting statistics of the data does not allow for a quantitative characterization of these substructures.

We examine the spectral properties of the key nonthermal features, individually. Fig. 8 shows the net spectrum of the halo, extracted from Region P0 (a local background described in $\S 2.4$ is subtracted). In addition to a dominant contribution from a power law with a photon index of $\Gamma \sim 1.7-2.1$, there is clearly a thermal component, as evidenced by the presence of the $\sim 1.34 \mathrm{keV} \mathrm{Mg}$ XI K $\alpha$ line. Characterized by a thermal plasma ( $k T \sim 0.6-1.2 \mathrm{keV}$ ), this component accounts for about $20 \%$ of the total emission from Region P0. The exact contribution from this thermal component is uncertain, somewhat depending on the background subtraction. The component, rich in magnesium $(\sim 0.6-4.5$ solar), may represent an enhanced surrounding thermal plasma emission projected along the line of sight.

Fig. 9 shows the net spectra of regions P1 (with the pulsar excluded) and P2-P8 (Fig. 4). The P1 spectrum is dominated by the SW-NE oriented bar $\left(\sim 2.4^{\prime \prime} \times 6.8^{\prime \prime}\right)$, which has a very high-surface brightness. We can thus divide the bar region into individual segments, but find no statistically significant spectral variation on either side of the pulsar. The spectrum of Region P1 can be well fitted $\left(\chi^{2} /\right.$ d.o.f. $\left.=206.3 / 191\right)$ by a power-law with a photon index of $2.18_{-0.53}^{+0.59}$ and a foreground absorption $N_{\mathrm{H}} \sim 6.1_{-2.4}^{+2.7} \times 10^{21} \mathrm{~cm}^{-2}$. The accumulated spectrum of the $\mathrm{P} 2-\mathrm{P} 8$ regions may also be characterized approximately $\left(\chi^{2} /\right.$ d.o.f. $\left.=318.1 / 241\right)$ by a power-law with an index of $2.62 \pm 0.04$ and a hydrogen column $N_{\mathrm{H}} \sim(6.3 \pm 0.2) \times 10^{21} \mathrm{~cm}^{-2}$. The $N_{\mathrm{H}}$ values are consistent with those inferred from the spectral analyses of the entire remnant $(\S 2.1)$ and the pulsar $(\S$ 2.2) . The spec- tra from the individual $\mathrm{P} 1-\mathrm{P} 8$ regions, fitted well with the power law, show a systematic change of the photon index along the major axis of the nebula (Fig. 10). Here we have fixed $N_{\mathrm{H}}$ to the value $6.1 \times 10^{21} \mathrm{~cm}^{-2}$; if $N_{\mathrm{H}}$ is allowed to vary in the fit, the result is very similar, albeit with larger error bars.

\subsection{Diffuse Emission: Thermal Component}

We characterize the properties of the diffuse X-ray emission away from the nonthermal cometary nebula by analyzing the ACIS-S spectra of individual regions of N157B (Fig. 14 Fig. 11). The spectrum of Regions L13 , excluding the background estimated in Regions B1 and $\mathrm{B} 2$, is used to characterize the general low-surface brightness emission from the SNR. For the spectral analysis of the substructures (D1, D2, and D3), we adopt the L1-3 spectrum as the local background. All these spectra show distinct emission lines, indicating predominant contributions from optically-thin thermal plasma. Indeed, the spectra can all be fitted well with the NEI model. Similar to the analysis of the accumulated spectrum from the entire SNR $(\S 2.1)$, the model fits allow the abundances of key line elements to vary, while leaving the rest of the metal abundances fixed to the LMC value (0.3 solar). Table 4 summarizes the net count rates and results of the spectral fits, as well as the inferred energy fluxes and hydrogen number densities of the individual regions. The densities are derived on the assumption that the 3-D shapes of the elliptical regions (D1-D3) are oblate ellipsoids and those of the rectangular regions (L1L3) are cuboids with the average line-of-sight size to be the remnant radius. The deviation from this assumption and the non-uniformity of the X-ray-emitting plasma are consolidated into the "filling factor," $f$, of the individual regions. The regions show significant spectral variation among them. While the metal abundances in Regions L1-3 are consistent with the LMC value, the substructures all exhibit significant metal enrichment and variations in physical conditions. For example, Region D3 shows both a strong O VIII K $\alpha$ line and a low plasma temperature, which explains the softness of its emission (e.g., Fig. 1b). Regions D1 and D2 show comparable temperatures, densities, and neon abundances, but Region $\mathrm{D} 1$ is also particularly abundant in $\mathrm{Mg}$, seen in both the spectrum and the EW map, and its ionization timescale is significantly smaller than that of Region D2.

It is possible that part of the diffuse X-ray emission may have origins other than the N157B SNR. However, there is little evidence for a spatial correlation of the diffuse X-ray intensity with stellar concentrations (e.g., Fig. 1), indicating that the direct stellar emission and the gas heating due to stellar winds from massive stars (e.g., wind-wind and wind-cloud collisions) do not contribute significantly. The X-ray emission from the preexisting superbubble is substantially softer than what is inferred from the SNR and has a very low-surface brightness (Smith \& Wang 2004). Our analysis is based on the enhanced emission above the local background, which includes the superbubble contribution.

\section{DISCUSSION}

Based on our analysis, we now attempt to present a coherent interpretation of the characteristics of N157B: 

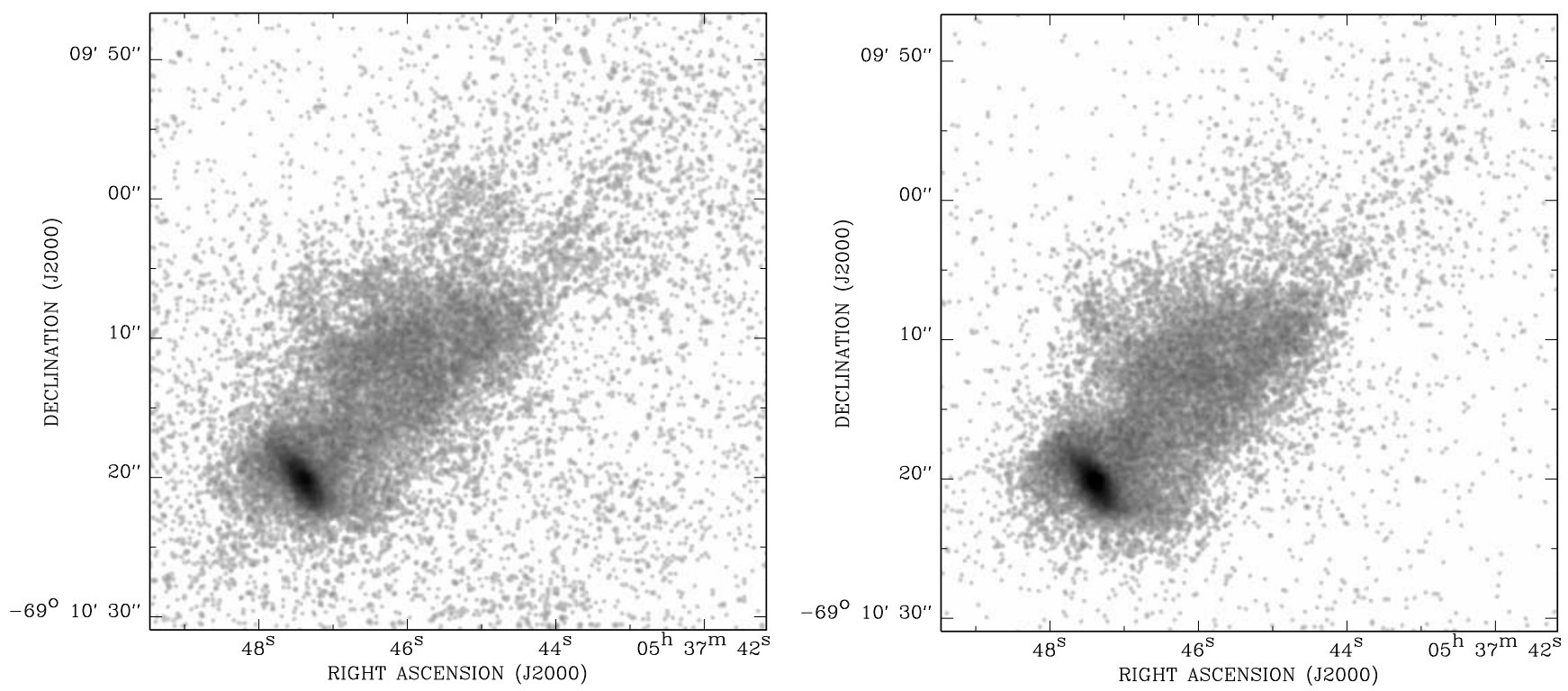

FIG. 7. - Close-ups of the PWN in the $0.3-1.5 \mathrm{keV}$ band (a) and the $1.5-7 \mathrm{keV}$ band (b). These raw count distributions, smoothed with a Gaussian with FWHM=0!'3, are plotted logarithmically.

TABLE 4

Spectral Fits for Individual Thermal Features

\begin{tabular}{|c|c|c|c|c|}
\hline parameter & L1-3 & D1 & D2 & D3 \\
\hline net count rate $\left(10^{-2}\right.$ counts s $\left.^{-1}\right)$ & $1.79 \pm 0.10$ & $4.06 \pm 0.11$ & $3.70 \pm 0.10$ & $3.76 \pm 0.13$ \\
\hline$\chi^{2} /$ d.o.f. & $23.6 / 24$ & $84.3 / 60$ & $87.1 / 62$ & $45.4 / 40$ \\
\hline$N_{\mathrm{H}}\left(10^{21} \mathrm{~cm}^{-2}\right)$ & $3.8 \pm 0.6$ & $7.4_{-0.4}^{+1.0}$ & $4.8_{-0.7}^{+0.8}$ & $9.3_{-2.8}^{+1.6}$ \\
\hline$k T(\mathrm{keV})$ & $1.04_{-0.31}^{+0.89}$ & $1.47_{-0.40}^{+0.59}$ & $1.22_{-0.31}^{+0.41}$ & $0.23_{-0.07}^{+0.8}$ \\
\hline$[\mathrm{O} / \mathrm{H}]$ & $\ldots$ & $\ldots$ & $\ldots$ & $0.79_{-0.40}^{+1.29}$ \\
\hline$[\mathrm{Ne} / \mathrm{H}]$ & $\cdots$ & $1.38_{-0.30}^{+0.36}$ & $0.96 \pm 0.26$ & $0.51_{-0.20}^{+0.50}$ \\
\hline$[\mathrm{Mg} / \mathrm{H}]$ & $\ldots$ & $2.23_{-0.84}^{+1.10}$ & $0.50_{-0.15}^{+0.31}$ & \\
\hline$n_{e} t_{i}\left(10^{10} \mathrm{~cm}^{-3} \mathrm{~s}\right)$ & $5.33_{-3.47}^{+8.30}$ & $0.68_{-0.15}^{+0.45}$ & $2.39_{-0.96}^{+0.93}$ & $2.30_{-1.38}^{+8.13}$ \\
\hline$f n_{e} n_{\mathrm{H}} V\left(10^{57} \mathrm{~cm}^{-3}\right)$ & $3.20_{-1.15}^{+2.05}$ & $7.33_{-2.50}^{+4.45}$ & $4.76_{-1.44}^{+2.50}$ & $540(<3460)$ \\
\hline flux $\left(10^{-13} \mathrm{ergs} \mathrm{cm}^{-2} \mathrm{~s}^{-1}\right)$ & 2.0 & 14.5 & 6.0 & 313 \\
\hline$n_{\mathrm{H}} f^{1 / 2}\left(\mathrm{~cm}^{-3}\right)^{\mathrm{a}}$ & 0.22 & 0.56 & 0.67 & 3.8 \\
\hline
\end{tabular}

Note. - The fluxes are in the $0.5-10 \mathrm{keV}$ band.

a In the estimate of the densities, we assume cuboidal volumes for rectangular regions L1-L3, with sizes $8.0 \times 7.5 \times 12 \mathrm{pc}^{3}, 14 \times 5.2 \times 12 \mathrm{pc}^{3}$, and $7.3 \times 2.9 \times 12 \mathrm{pc}^{3}$, respectively, and oblate spheroids for elliptical regions D1-D3, with half axes $8.6 \times 8.6 \times 2.2 \mathrm{pc}^{3}, 5.9 \times 5.9 \times 2.1 \mathrm{pc}^{3}$, and $7.2 \times 7.2 \times 5.0 \mathrm{pc}^{3}$, respectively.

the exceptionally large sizes of both the thermal and nonthermal components and the lack of a well-defined outer shell as well as the distinct spatial/spectral substructure and metal enrichment pattern.

\subsection{Environmental effect}

N157B is presumably associated with the OB association LH99, as they have comparable foreground absorption/extinction (WG98), in addition to their apparent superposition in the sky. While the characteristics of N157B appear to be a manifestation of its association with LH99, the new X-ray results provide new insights into the nature of the SN progenitor and the properties of the ambient environment. First, we can use the observed pattern of metal enrichment to constrain the mass of the progenitor star. As the presence of a pul- sar implies a core-collapse nature of the SN, we plot in Fig. 12 the relative metal abundances predicted from such SN models for stars with low metallicities (Thielemann, Nomoto, \& Hashimoto 1996, hereafter TNH96; Woosley \& Weaver 1995, hereafter WW95). The enrichment is compatible with the TNH96 model for a progenitor mass of $20-25 M_{\odot}$, or the WW95 models for a progenitor mass of $30-35 M_{\odot}, Z=0.1 Z_{\odot}$, and an explosion energy of $\sim 1.2 \times 10^{51} \mathrm{ergs}$. Although these two model predictions do not overlap, they both indicate a progenitor mass of $\gtrsim 20 M_{\odot}$. Recent models taking into account stellar mass loss suggest that neutron stars can be produced by metal-poor progenitors only if the progenitor mass is $<25 M_{\odot}$ (Heger et al. 2003). This mass limit, while consistent with the above estimate from the TNH96 model, is lower than the masses of the O3 and O5 


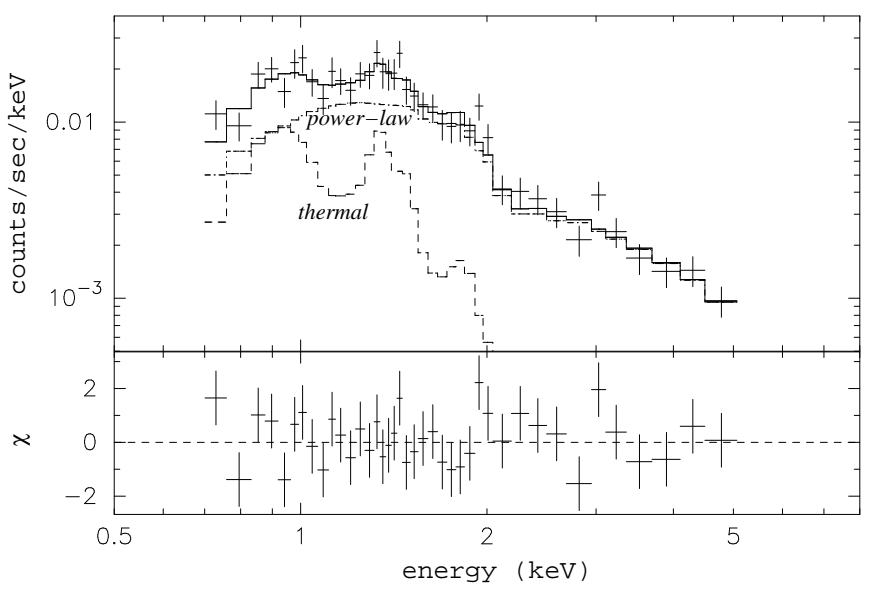

FIG. 8.- ACIS-S spectrum in the regions P0, fitted with a power-law plus an NEI thermal plasma.
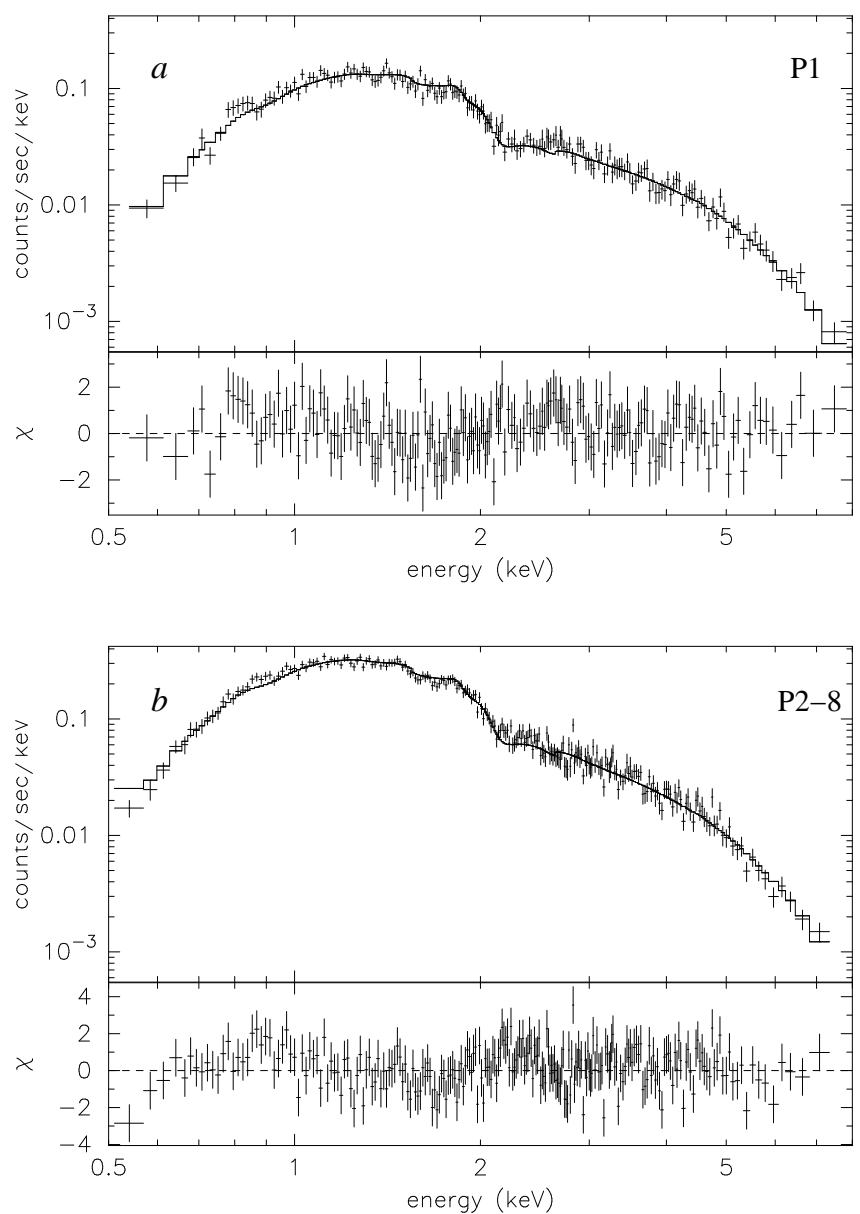

FIG. 9.- ACIS-S spectra in the Regions P1 (a) and P2-P8 (b), together with power-law fits.

stars that still exist in LH99 (Schild \& Testor 1992). The earlier explosion of a less massive star thus implies that the star formation in the vicinity of LH99 is not coeval. Further evidence for a prolonged star formation has recently been provided by the massive proto-stars revealed in Spitzer observations; for example, the brightest point source in the Spitzer $8 \mu \mathrm{m}$ image is a proto-star (Fig. प $\mathrm{a}$ ).

We next examine how the co-existence with the $\mathrm{OB}$

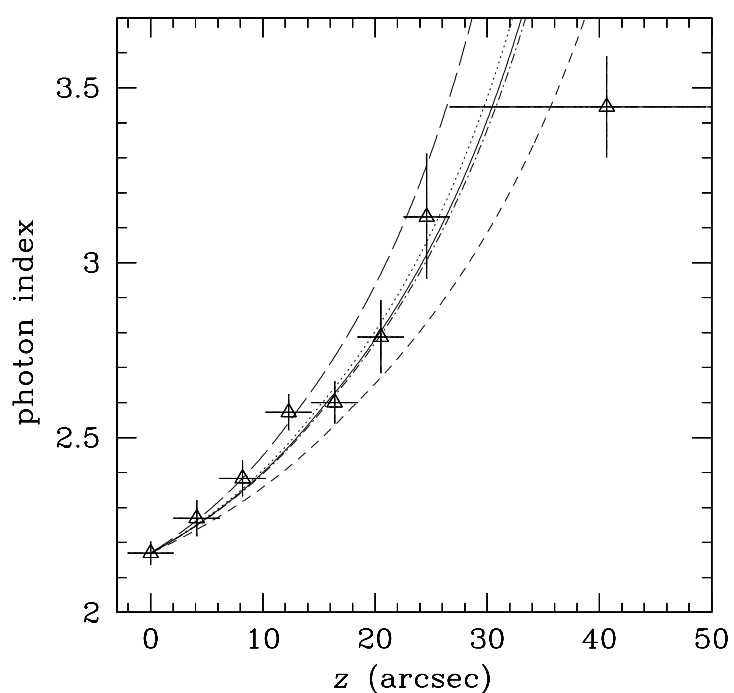

FIG. 10.- Variation of the power-law photon index of the nonthermal tail as a function of the distance from the pulsar. Curves are plotted according to the particle ejection model represented by Eq. 10] with various $u$ and $B_{\perp}$ parameter combinations: $0.5 \mathrm{c}$ and $0.9 \times 10^{-4} \mathrm{G}$ (dotted curve), $0.6 c$ and $0.9 \times 10^{-4} \mathrm{G}$ (short-dashed), $0.6 c$ and $1.0 \times 10^{-4} \mathrm{G}$ (solid), $0.6 c$ and $1.1 \times 10^{-4} \mathrm{G}$ (long-dashed), and $0.7 c$ and $1.1 \times 10^{-4} \mathrm{G}$ (dot-dashed).

association LH99 may have influenced the evolution of N157B. Fig. 17 shows a Spitzer IRAC $8 \mu \mathrm{m}$ view of the large-scale environment of N157B. The $8 \mu \mathrm{m}$ band image is dominated by emission from polycyclic aromatic hydrocarbons (PAHs). As PAHs can be easily dissociated by UV radiation from $\mathrm{O}$ stars, the $8 \mu \mathrm{m}$ emission traces $\mathrm{H}$ I gas just outside the ionization front. The $8 \mu \mathrm{m}$ image shows that N157B is inside a large cavity that might be a superbubble produced by the mechanical energy input from the OB association LH99. The existence of a blister-type superbubble around N157B has also been suggested previously based on the velocity structure of dense ionized gas (Chu et al. 1992). This IR cavity might also be connected with the cavities and "tunnels" powered by multiple OB associations, including also LH90 to the east, as indicated by the presence of a large-scale $\left(\sim 20^{\prime}\right)$ low-surface brightness X-ray emission in the region (Smith \& Wang 2004). The exact shape of the midinfrared cavity, let alone its boundaries, is very uncertain; there may be substantial projection effect. In morphology, the pulsar, presumably close to the explosion center, is located at the west edge of the cavity. The large-scale diffuse X-ray emission extends into the cavity. The expansion of the SNR into such a low-density hot medium at least partly explains its unusual large size and its lack of a well-defined outer shell. The hot ambient medium has a high isothermal sound velocity, thus the shocks are expected to be weak with small Mach numbers, and the blastwave may become thermalized and deposit the bulk of the SNR energy into the thermal energy of the hot medium (Tang \& Wang 2005). The relatively low pressure inside the SNR further allows for the expansion of the PWN to an exceptionally large volume.

While the overall morphology of N157B is apparently affected by the large-scale superbubble environment, the 

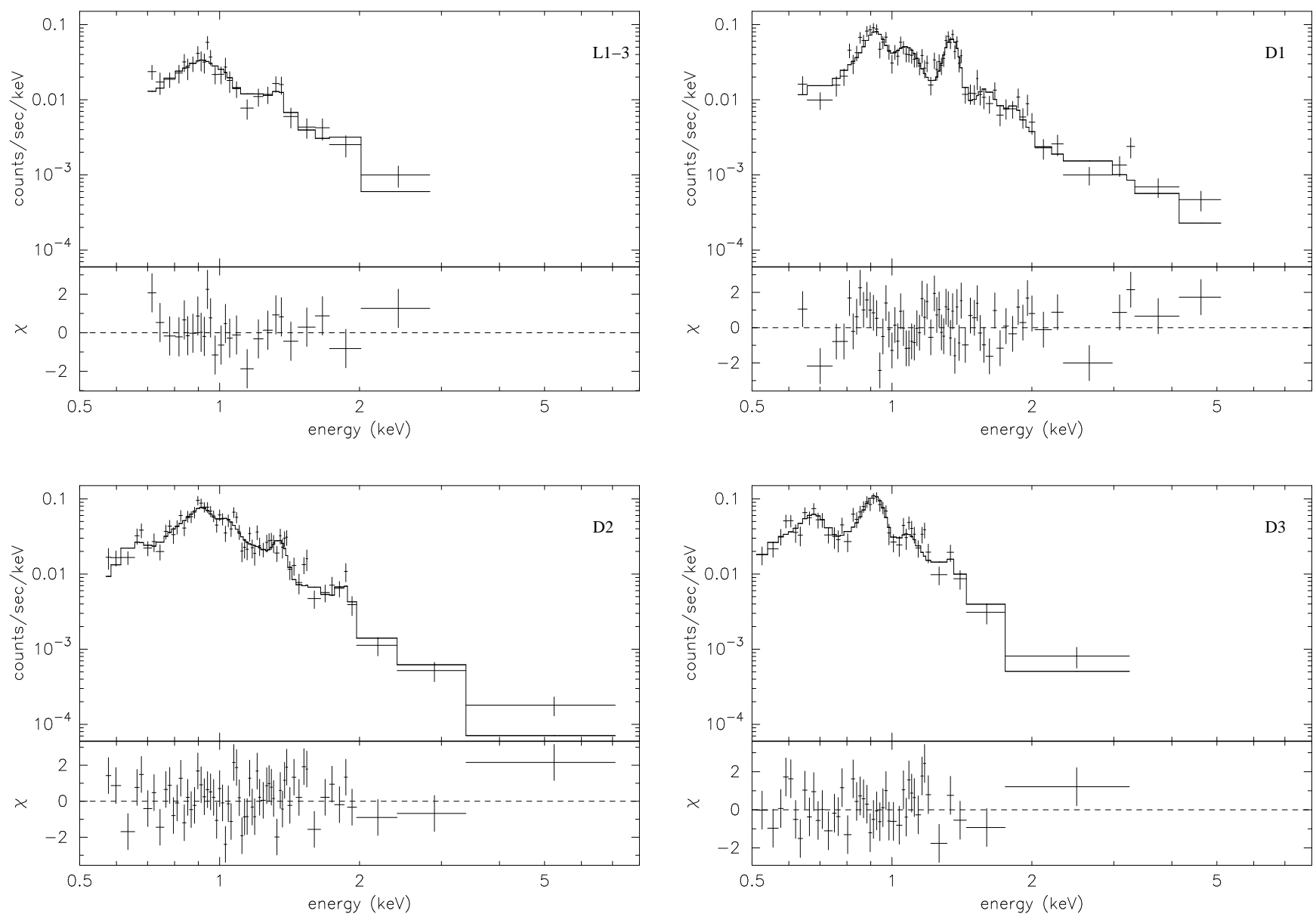

Fig. 11. - ACIS-S spectra of selected thermal substructures (see Fig. 4 L1-3, D1, D2, and D3). All these spectra are fitted with the NEI model (Table 4 and are re-binned to achieve a background-subtracted $\mathrm{S} / \mathrm{N}$ ratio of 3 .

presence of a dense cloud close to the explosion site seems to play a critical role in shaping the dynamics of the materials around the pulsar. This provides an alternative explanation for the trailing PWN structure, which we previously proposed to be due to pulsar motion (WG98; Wang et al. 2001). Probably, the trailing tail is not necessarily produced by the passage of the moving pulsar; and an existing example may be the Duck X-ray Nebula, where the tail is ejection of pulsar wind particles at weak relativistic bulk velocity confined by the ambient medium ram-pressure (Kaspi et al. 2001). In SNR N157B, the pulsar appears to be near the explosion center and have not moved out of the SNR, at least in projection. The size of the nonthermal nebula, especially its head, is large, which is somewhat difficult to explain in a supersonic relative motion scenario, although the physics of the shocked pulsar wind is quite uncertain, complicated by its non-spherical symmetry. Instead, the subsonic ram-pressure confinement can be consistent with the gas motion relative to the pulsar (see below and 3.2 . The cloud is partly seen as the strongest diffuse mid-infrared emission peak south to the pulsar in Fig. 1. The cometary PWN tail points to the northwest, almost parallel to the long dimension of the cloud or the southwest edge of the superwind cavity (Fig.1a), consistent with the relatively narrow extent of the thermal emission on the southwest side of the PWN (Fig.1b and
Fig.1d). The northern part of the cloud, not apparent in Fig. 11, is bright in optical (Fig. 18). The intensity of the enhanced diffuse optical light shows a reasonably good morphological similarity to the relatively high surfacebrightness X-ray emission in the $0.3-1.5 \mathrm{keV}$ band. The dust lane at the southeast side of Fig. 11 casts a shadow in the emission distribution and corresponds to harder X-ray emission (Fig. 10), apparently due to the X-ray absorption (but the limited counting statistics and the uncertainty in the background subtraction make it hard to quantify the absorbing gas column). In fact, the association of N157B with this optical nebula has already been suggested by Chu et al. (1992), based on its kinematics. In view of these observational factors, we suggest a physical association that is produced by the impact of N157B on the cloud. Although the shape and boundary of the mid-infrared cavity are very uncertain (considering substantial projection effect) and the exact interplay (the structure and dynamics) between the two cannot yet be determined, this impact likely led to a strong reflection shock, which has probably swept through and been further reflected around much of the PWN. It is also possible that there is a reflection from the east edge of the cavity, although we find little optical evidence for a strong interaction. The resultant heating by reflection shock and possibly large bulk flow of the SNR gas may thus be responsible for both the observed one- 
sided morphology of the PWN and the thermal emission enhancement (like a sheath) around the southern part of the PWN. The enhancement east of the pulsar (Region D2) is particularly strong just $\left(\sim 10^{\prime \prime}-20^{\prime \prime}\right)$ east of the pulsar and is bordered by a dust lane farther to the east. It shows no evidence for nonthermal emission. This part of the enhancement is clearly edge-enhanced and has corresponding optical brightening (Fig. 11), probably representing the strongest ongoing reflection of the SNR from the cloud. The high-pressure of the reflected gas may have greatly influenced the confinement of the pulsar wind and hence the direction of its outflow toward the northwest. This interpretation of the PWN and its surrounding medium is similar to the scenarios proposed by WG98 and van der Swaluw et al. (2003): the observed one-sided morphology is a result of an asymmetric rampressure confinement. But, the present interpretation of the strongly one-sided PWN morphology does not require a fast proper motion of the pulsar; the ram-pressure (or the relative motion between the pulsar and the surrounding medium) can naturally arises from the highly asymmetric reflection shock of the SNR (see more discussion below). Having proposed this alternative, we do not abandon the pulsar proper motion scenario, which is still plausible.

\subsection{Evolutionary Stage of SNR N157B}

The properties of N157B is determined not only by its environment but also by its specific evolutionary stage, although its age is still very uncertain. The spindown age of the associated pulsar PSR J0537-6910 is $\sim 5 \times 10^{3} \mathrm{yr}$, as derived from the pulse period and its derivative, assuming a negligible initial pulsar period and a dipole magnetic field configuration (Marshall et al. 1998). These assumptions may not be valid for young pulsars in general and for this pulsar in particular because it displays repeated episodes of strong timing glitches (Marshall et al. 2004). Nevertheless, the spin-down age is roughly consistent with the large size of the remnant (12 pc in radius for a total angular extent of $\left.\sim 100^{\prime \prime}\right)$. From the ionization timescale $\left(n_{e} t_{i} \sim 2.4 \times 10^{10} \mathrm{~cm}^{-3} \mathrm{~s}\right.$; Table 3) and the mean gas density $\left(n_{\mathrm{H}} \sim 0.45 f^{-1 / 2} R_{12}^{-1 / 2} \mathrm{~cm}^{-3}\right.$ where $R_{12}=R / 12 \mathrm{pc}$, assuming $n_{e} \sim 1.2 n_{\mathrm{H}}$ ), we may estimate an average ionization age of the X-ray-emitting plasma in the remnant (i.e., the time elapsed since it was shocked) as $t_{i} \sim 2 \times 10^{3} f^{1 / 2} R_{12}^{1 / 2} \mathrm{yr}$, which is considerably shorter than the pulsar spin-down age, especially because $f$ can be small. This discrepancy may be understood if the Xray emission originates primarily from the plasma that is recently shock-heated.

Indeed, the thermal properties of N157B, as inferred from $\S 2.4$, suggest that the X-ray-emitting gas consists of various metal-enriched clumps embedded in a more diffuse plasma. This diffuse plasma is distributed more widely than the clumps and also seems to have both a nominal LMC metal abundance and a larger average ionization age, which is comparable to the spin-down age of the pulsar. Therefore, the plasma may mostly represent the shocked ISM, whereas the clumps are mainly due to SN ejecta. Unfortunately, the quality of the data is not adequate to make a quantitative decomposition of these two components. We could only make a crude estimate of the thermal energy as $\sim 3 \times 10^{50} f^{1 / 2} R_{12}^{3 / 2}$ ergs and the mass as $\sim 110 f^{1 / 2} R_{12}^{3 / 2} M_{\odot}$. This estimate is subject to the uncertainty in the effective filling factor, which cannot be determined and is small, probably on the order of $\lesssim 0.1$. This gas mass is comparable to several solar masses expected for the SN ejecta and is thus consistent with the significant metal-enrichment detected. Depending on the $f$ value, the thermal energy may be considerably smaller than the canonical SN energy $\left(\sim 10^{51} \mathrm{ergs}\right)$. A substantial fraction of the energy may still be in the kinetic form, carried primarily by a weak outward blastwave into the low-density hot superbubble interior $(\S 3.1$; Tang \& Wang 2005).

Among the substructures considered, the enhancement in Region D3 shows distinct characteristics: a partially round shape with a size of $\sim 30^{\prime \prime}$ or $7 \mathrm{pc}$, a distinctly low gas temperature $(\sim 0.2 \mathrm{keV})$, a high density $\left(\sim 4 f^{-1 / 2} \mathrm{~cm}^{-3}\right)$, a low ionization age $\left(\sim 160 f^{1 / 2} \mathrm{yr}\right)$, and an apparent overabundance in oxygen. This feature may represent a clump of oxygen-rich ejecta recently heated by a low-velocity reverse shock $\left(\sim 400 \mathrm{~km} \mathrm{~s}^{-1}\right)$, as inferred from the gas temperature. However, this hypothesis cannot explain the morphology or size, as the ionization time scale (Table 4) would suggest a velocity of $\sim 4 \times 10^{4} f^{-1 / 2} \mathrm{~km} \mathrm{~s}^{-1}$, much higher than the value inferred from the plasma temperature. Of course, the NEI plasma we used may be an over-simplification for the modeling of the spectrum (Fig. 111), and the inferred ionization time scale may be misleading. Without the constraint on the time scale, the D3 enhancement may simply be part of the shocked SN ejecta, possibly ballooning out from the high-pressure SNR interior into the superbubble. In this case, the relatively low temperature can be naturally explained by the adiabatic cooling of the expanding gas. Alternatively, D3 may arise from a separate oxygen-rich $\mathrm{SN}$, which is quite possible with the presence of the OB association LH99. In this case, the partially round morphology can naturally be explained as a coherent shell of reverse-shocked SN ejecta. The age of the remnant should be comparable to the ionization age inferred above. The reverse shock velocity is expected to be substantially lower than that of the forward blastwave, which is probably very weak in X-ray emission. If the remnant is also expanding inside the superbubble, the lack of an apparent radio or optical counterpart is then expected. The absence of a PWN associated with the remnant further suggests that the stellar progenitor of the SN is more massive ( $\gtrsim 25 M_{\odot}$; Heger et al. 2003) and that the stellar remnant may be a black hole. In fact, we are also a bit uncomfortable about involving a second SNR in the interpretation. But if we accept the spectral results (mainly the small ionization parameter) from the NEI model fit to the D3 spectrum, we find no better solution than a new SNR.

As discussed in $\S 3.1$, a "sheath" of the thermal Xray enhancement (including D1+D2) around the PWN is likely a result of its interaction with the material reflected off the dense cloud. The physical processes involved are similar to those in the interaction between an SNR reverse shock and a PWN of a fast moving pulsar, as simulated by van der Swaluw et al. (2004). Before such an interaction, the PWN consists of two main components, the shocked pulsar wind material and the swept-up SN 


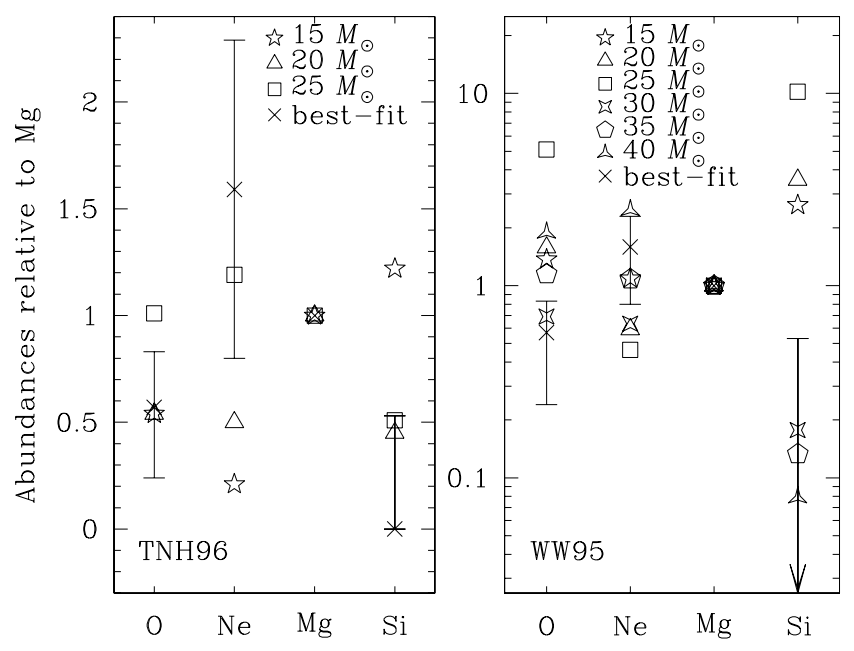

FIG. 12.- The best-fit abundances relative to $\mathrm{Mg}$, compared with the predictions from core-collapsed SN models (TNH96 and WW95). Note that because the best-fit Si value is only $\sim 10^{-8}$, it is invisible in the right panel (with a logarithmic scale).

ejecta between the forward shock and the contact discontinuity (Reynolds \& Chevalier 1984). The thermal emission from the swept-up SN ejecta is expected to be weak and is confined within a relatively thin shell. The passage of the strong reflection shock (e.g., stage 2 in van der Swaluw et al. 2004) compresses the PWN, sweeps part of it into the trailing tail, and is meanwhile further reflected forward. Consequently, both the previous undisturbed and the swept-up SN ejecta are compressed and heated at the front side of the PWN. The density and temperature in this "sheath" around the PWN should be enhanced by a factor of 1-2.5 (compared to those in the upstream gas), depending on the Mach number of the reflection shock relative to the pulsar (Spitzer 1982; Hester \& Cox 1986). Such density and temperature contrasts are consistent with our measurements, albeit with large uncertainties (Table 4). We thus conclude that the interaction between the reflected material and the PWN provides a natural interpretation of both its strongly one-sided morphology and its surrounding thermal X-ray emission enhancement.

\subsection{Physical Properties of the PWN}

Based on the spectroscopic results of various nonthermal components of N157B, we attempt to determine their nature and quantify their physical parameters. The bar around the pulsar PSR J0537-6910 has been proposed to be a torus (Wang et al. 2001), similar to those detected around several well-known young pulsars [e.g., Crab (Weisskopf et al. 2000), Vela (Helfand, Gotthelf, \& Halpern 2001), PSR 0540-69 (Gotthelf \& Wang 2000), and $\mathrm{G} 54.1+0.3$ (Lu et al. 2002)] and believed to represent the termination shocks of the toroidal pulsar winds (e.g., Ng \& Romani 2004). This proposal is consistent with the results from our spectral analysis of the bar. The lack of a systematic spectral variation across the bar simply reflects similar particle acceleration and synchrotron emission throughout the termination shock region, just as in the Crab nebula (Mori et al. 2004). The power-law photon index inferred from the analysis also agrees well with the values $(\sim 2)$ observed in the other toruses (Gotthelf 2003). The centrally enhanced bar-like morphology can be naturally explained by the edge-on orientation of the torus because of the expected Doppler boosting, which depends on the angle between the bulk motion velocity of shocked pulsar particles and our sight line (e.g., Komissarov \& Lyubarsky 2003). Similar effects have been observed in both the Crab nebula and G54.1+0.3 (Lu et al. 2002). The size of the torus should be comparable to the radius of the termination shock (modified from Chevalier 2000),

$$
r_{t}=\left(\frac{6 \varepsilon_{B} \dot{E}}{c \sin \theta_{\mathrm{t}}}\right)^{1 / 2} B^{-1}
$$

where $\varepsilon_{B}$ denotes the magnetic field fraction of the pulsar wind luminosity ( $\sim 0.5$ in the equipartition case) and $\theta_{\mathrm{t}}$ is the half angular span of the toroidal wind (i.e., $4 \pi \sin \theta_{\mathrm{t}}$ is the solid angle subtended at the pulsar by the torus). The pulsar spin-down power $\dot{E}=4.8 \times 10^{38} \mathrm{ergs} \mathrm{s}^{-1}$ (Wang et al. 2001; Mignani et al. 2005) is adopted. The magnetic field $B$ just behind the shock is not well understood. But if it is comparable to the average strength $\left(\sim 2 \times 10^{-4} \mathrm{G}\right)$ in the PWN and $\theta_{\mathrm{t}}=20^{\circ}$ (as estimated in Wang et al. 2001), we can then estimate $r_{t} \approx 0.6 \mathrm{pc}$ or 2 ". 4. This estimate is comparable to the observed overall dimension $(\sim 2$.! $4 \times 6$.! 8$)$ of the bar, and thus both the morphological and spectral characteristics of the bar are consistent with its being an edge-on torus resulting from the terminal shock of the pulsar toroidal wind.

The N157B PWN as a whole shows a featureless and hard spectrum. The spectral steepening along the major axis of the tail indicates significant synchrotron cooling. Similar spectral properties have been seen in other SNRs, such as G21.5-0.9 (Slane et al.. 2000; Warwick et al. 2001, Safi-Harb et al. 2001), 3C58 (Torii et al. 2000), IC443 (Bocchino \& Bykov 2001), and possibly in W44 (Petre, Kuntz, \& Shelton 2002); however, their X-ray softening has not been quantitatively compared with models. The bright and long trailing pattern of the N157B nebula makes it possible to quantitatively compare observations with models.

We construct a simple 1-D toy model, similar to the spherically symmetrical treatment by Amato et al. (2000). In the 1-D approximation of the N157B PWN, the shocked wind particles stream off the bar along the downstream tail at a speed $u$ within a cylinder of radius $r$. The differential particle density distribution as a function of the distance from the injection point (the bar) $z$ is then

$$
N(E, z)=\frac{J\left(E_{\text {ini }}\right)}{\pi r^{2}}\left|\frac{\partial t_{\text {ini }}}{\partial z}\right|\left|\frac{\partial E_{\text {ini }}}{\partial E}\right|,
$$

where $E_{\text {ini }}$ and $t_{\text {ini }}$ are the initial energy and time of the injected particle, while the injection rate $J\left(E_{\text {ini }}\right)$ is assumed to be a power law,

$$
J\left(E_{\text {ini }}\right)=K E_{\text {ini }}^{-p}
$$

If the bulk velocity $u$ is assumed to be constant (i.e., $z / t$ ), the energy loss of the particles resulting from the synchrotron radiation is then

$$
\frac{d E}{d t}=-c_{1} B_{\perp}^{2} E^{2}
$$


where $c_{1}=4 e^{4} /\left(9 m_{e}^{4} c^{7}\right)$ and $B_{\perp}$ is the magnetic field component perpendicular to the particle motion. Integration of the above equation gives $E_{\mathrm{ini}}=E /\left(1-E / E_{\mathrm{m}}\right)$, where

$$
E_{\mathrm{m}}=u /\left(c_{1} B_{\perp}^{2} z\right)
$$

marking the maximum energy of the particles after the synchrotron loss at $z$. Then the particle density distribution is given by

$$
N(E, z)=\frac{K}{\pi r^{2} u} E^{-p}\left(1-\frac{E}{E_{\mathrm{m}}}\right)^{p-2} .
$$

The synchrotron emissivity of the particles is $j_{\epsilon}=$ $\left(c_{1} B_{\perp}^{2} E^{2}\right) N(E, z) d E / d \epsilon$, where the photon energy is related to the particle energy as $\epsilon=c_{2} h B_{\perp} E^{2}$ [where $\left.c_{2}=0.29 \times 3 e /\left(4 \pi m_{e}^{3} c^{5}\right)\right]$. Therefore

$$
j_{\epsilon}=\frac{c_{1}}{2 c_{2} h} \frac{K}{\pi r^{2} u} B_{\perp} E^{1-p}\left(1-\frac{E}{E_{\mathrm{m}}}\right)^{p-2} .
$$

The dependence of the emission's photon index,

$$
\Gamma \equiv-\left(1+d \ln j_{\epsilon} / d \ln \epsilon\right),
$$

implicitly on $\epsilon$ and $z$ (via $E$ and $E_{\mathrm{m}}$, respectively) is derived as

$$
\Gamma=\frac{p+1}{2}+\frac{p-2}{2} \frac{E / E_{\mathrm{m}}}{1-E / E_{\mathrm{m}}}
$$

The above equation shows that the spectral index varies with both the photon energy and the distance from injection point. Since the spectral indices are determined in a broad energy range, to compare with the observed values, we calculate a weighted spectral index, $\bar{\Gamma}=\int_{\varepsilon_{1}}^{\min \left(\varepsilon_{\mathrm{u}}, \varepsilon_{\mathrm{m}}\right)}$ $[j(\nu, z) / \epsilon] \Gamma(\nu, z) d \epsilon / \int[j(\nu, z) / \epsilon] d \epsilon$, namely

$\bar{\Gamma}=\frac{p+1}{2}+\frac{p-1}{2} \frac{\left(\sqrt{\varepsilon_{\mathrm{m}} / \varepsilon_{1}}-1\right)^{p-2}-\left(\sqrt{\varepsilon_{\mathrm{m}} / \min \left(\varepsilon_{\mathrm{u}}, \varepsilon_{\mathrm{m}}\right)}-1\right)^{p-2}}{\left(\sqrt{\varepsilon_{\mathrm{m}} / \varepsilon_{1}}-1\right)^{p-1}-\left(\sqrt{\varepsilon_{\mathrm{m}} / \min \left(\varepsilon_{\mathrm{u}}, \varepsilon_{\mathrm{m}}\right)}-1\right)^{p-1}} 3$

where $\varepsilon_{1}$ and $\varepsilon_{\mathrm{u}}$ are the lower and upper energy limits of the spectrum used, while $\varepsilon_{\mathrm{m}}$ is the photon energy corresponding to $E_{\mathrm{m}}$,

$$
\varepsilon_{\mathrm{m}}=\left(\frac{z}{17 \mathrm{pc}}\right)^{-2}\left(\frac{u}{c}\right)^{2}\left(\frac{B_{\perp}}{10^{-4} \mathrm{G}}\right)^{-3} \mathrm{keV} .
$$

Eq. 10 can be compared with the measured values. We fix the photon index at the injection point $(\mathrm{P} 1)$, $\Gamma=2.17\left(\right.$ for $\left.N_{\mathrm{H}}=6.1 \times 10^{22} \mathrm{~cm}^{-2}\right)$, which corresponds to $p=3.34$, and adopt $\varepsilon_{1}=0.5 \mathrm{keV}$ and $\varepsilon_{\mathrm{u}}=7 \mathrm{keV}$. Fig. 10] shows predicted curves, assuming the magnetic field to be around the mean PWN value (Wang et al. 2001). These curves match the measured values well, suggesting $u \gtrsim 0.5 c$, comparable to the sound speed of an ultra-relativistically hot plasma $\left(c_{s}=c / \sqrt{3}\right)$. Similar high downstream flow speeds are observed or inferred at the terminal shocks of the Crab nebula (Hester et al. 2002) and G54.1+0.3 (Lu et al. 2002); but in these cases, the flows are presumably quickly damped and energy is then transported primarily due to particle diffusion. In an one-sided pressure confined PWN, the downstream flow is re-directed into a bulk motion at a similar or higher speed, as demonstrated analytically (e.g.,
Wang, Li, \& Begelman 1993) and in simulations (e.g., Bucciantini et al. 2005). Therefore, a strong pressureconfinement of the PWN is needed to give a consistent interpretation of both the exceptionally large linear size and the observed spectral steepening of the nonthermal component of the N157B PWN.

\section{SUMMARY}

Based on our on-axis Chandra ACIS observation, we have conducted a detailed spatially-resolved X-ray spectroscopy of N157B. Combining the X-ray analysis with the optical and mid-infrared observations, we have explored the physical origins of various distinct characteristics and their implications. Our main results and conclusions are summarized as follows:

1. We detect eight point-like X-ray sources in the ACIS observation. Two of these sources in the vicinity of the pulsar PSR J0537-6910 have nearIR and optical counterparts, which are identified as massive stars in the LMC. The relatively high $\mathrm{X}$-ray luminosities of the two sources indicate that they are probably colliding stellar wind binaries. A comparison of the X-ray and 2MASS positions of the two sources suggests that the absolute astrometry of the ACIS observation, hence the X-ray centroid position of the pulsar, is better than about 0.5 .

2. We confirm the nonthermal nature of the various high-surface brightness X-ray features, such as the bar, tail, and halo, as suggested in the previous morphological and integrated spectral studies. The bar shows no significant spectral shape variation and most likely represents the terminal shock of a toroidal pulsar wind. The strong spectral steepen-2 ing observed in the tail is consistent with the synchrotron radiation loss and suggests a bulk velocity

3. The thermal emission is unambiguously resolved to arise from a region much larger than the nonthermal PWN and accounts for about one-third of the total X-ray emission in the $0.5-10 \mathrm{keV}$ band. The thermal emission consists of various clumps embedded in a diffuse X-ray-emitting plasma. The clumps are over-abundant in $\alpha$ elements and thus represent the SN ejecta. The over-abundance pattern suggests a mass $\gtrsim 20 M_{\odot}$ for the SN progenitor. However, a partially-round-shaped soft X-ray clump in the outer region of N157B shows distinct spectral characteristics, unusually low in both temperature and ionization time scale and overabundant in oxygen. This clump may thus represent a separate young SNR.

4. The thermal emission of N157B is enhanced around the nonthermal PWN, particularly near its head. This enhancement is perhaps a result of the recent compression and heating of the ejecta due to the SNR reflection from a nearby dense cloud. This interaction of the SNR reflection and the PWN naturally explains its one-sided morphology.

5. The relatively weak thermal emission, the lack of a rim-brightened outer blastwave, and the large sizes 
of both the thermal and nonthermal components all point to a scenario that SNR N157B is expanding into a low-density hot medium, which likely represents the interior of a superbubble created by the co-existing $\mathrm{OB}$ association.

We thank the referee Leisa Townsley for a thorough review of this work and for useful comments that led to various improvements in the presentation of the paper. We also thank Yang Su and Jiang-Tao Li for technical assistance. Y.C. acknowledges support from NSFC grants 10073003 and 10221001 and grant nkbrsf-g19990754 of the China Ministry of Science and Technology, while Q.D.W. acknowledges the support from NASA/CXC under the grant NAG5-3073A. This publication makes use of data products from the Two Micron All Sky Survey, which is a joint project of the University of Massachusetts and the Infrared Processing and Analysis Center/California Institute of Technology, funded by the National Aeronautics and Space Administration and the National Science Foundation. We also acknowledge the use of the SIMBAD and NED databases.

\section{REFERENCES}

Amato, E., Salvati, M., Bandiera, R., Pacini, F., \& Woltjer, L. 2000, A\&A, 359, 1107

Bocchino, F. \& Bykov, A. M. 2001, A\&A, 376, 248

Borkowski, K. J., Lyerly, W. J., \& Reynolds, S. P. 2001, ApJ, 548, 820

Bucciantini, N., Amato, E., \& Del Zanna, L. 2005, A\&A, 434, 189

Chevalier, R. A., 2000, ApJ, 539, 45

Chu, Y.-H., Kennicutt, R. C., Jr., Schommer, R. A., \& Laff, J. 1992, AJ, 103, 1545

Crawford, F., McLaughlin, M., Johnston, S., Romani, R., \& Sorrelgreen, E. 2005, AdSpR, 35, 1181

Davis, J. E. 2001, ApJ, 562, 575

Dennerl, K., et al. 2001, A\&A, 365, L202

Fazio, G. G., et al. 2004, ApJS, 154, 10

Feigelson, E., et al. 2002, ApJ, 574, 258

Frail, D. A., Giacani, E. B., Goss, W. M., \& Dubner, G. 1996, ApJ, 464, L165

Gaensler, B. M., Chatterjee, S., Slane, P. O., van der Swaluw, E., Camilo, F., Hughes, J. P. 2006, ApJ, in press, (astro-ph/0601304)

Gotthelt, E. V., 2003, ApJ, 591, 361

Gotthelf, E. V. \& Wang, Q. D. 2000, ApJ, 532, 117

Heger, A., Fryer, C. L., Woosley, S. E., Langer, N., Hartmann, D. H. 2003, ApJ, 591, 288

Helfand, D. J., Gotthelf, E.V., \& Halpern, J. P. et al. 2001, ApJ, ApJ, 556, 380

Henize, K, G. 1956, ApJS, 2, 315

Hester, J. J., Mori, K., Burrows, D., Gallagher, J. S., Graham, J. R., Halverson, M., Kader, A., Michel, F. C., Scowen, P. 2002, ApJ, 577, L49

Hwang, U., Holt, S. S., \& Petre, R. 2000, ApJ, 537, L119

Jerius, D., Donnelly, R. H., Tibbetts, R. J., Edgar, R. J., Gaetz, T. J., Schwartz, D. A., Speybroeck, L. P., \& Zhao, P. 2000, Proc. SPIE, 4012, 17

Kaspi, V. M., Gotthelf, E. V., Gaensler, B. M., \& Lyutikov, M. 2001, ApJ, 562, L163

Komissarov, S. S. \& Lyubarsky, Y. E. 2003, MNRAS, 344, L93

Lazendic, J. S., Dickel, J. R., Haynes, R. F., Jones, P. A., White, G. L. 2000, ApJ, 540, 808

Lu, F. J., Wang, Q. D., Aschenbach, B., Durouchoux, P., \& Song, L. M. 2002, ApJ, 568, L49

Lucke, P. B., \& Hodge, P. W. 1970, AJ, 75, 171

Marshall, F.E., Gotthelf, E.V., Zhang, W., Middleditch, J., \& Wang, Q. D. 1998, ApJ, 499, 179

Marshall, F.E., Gotthelf, E.V., Middleditch, J., Wang, Q. D., \& Zhang, W. 2004, ApJ, 603, 682

Matheson, H. \& Safi-Harb, S. 2005, AdSpR, 35, 1099.

Mignani, R. P., Pulone, L., Iannicola, G., Pavlov, G. G., Townsley, L., \& Kargaltsev, O. Y. 2005, A\&A, 431, 659
Mori, K; Burrows, D. N., Hester, J. J., Pavlov, G. G., Shibata, S., Tsunemi, H. 2004, ApJ, 609, 186

Morrison, R., \& McCammon, D., 1983, ApJ, 270, 119

Ng, C.-Y. \& Romani, R. W., 2004, ApJ, 601, 479

Olbert, C. M., Clearfield, C. R., Williams, N. E., Keohane, J. W., \& Frail, D. A., 2001, ApJ, 554, L205

Oskinova, L. M. 2005, MNRAS, 361, 679

Park, S., Roming, P. W. A., Hughes, J. P., Slane, P. O., Burrows, D. N., Garmire, G. P., \& Nousek, J. A., 2002, ApJ, 564, L39

Petre, R., Kuntz, K. D., \& Shelton, R. L. 2002, ApJ, 579, 404

Reynolds, S. P. \& Chevalier, R. A. 1984, ApJ, 278, 630

Russell, S. C. \& Dopita, M. A. 1992, ApJ, 384, 508

Safi-Harb, S., Harrus, I. M., Petre, R., Pavlov, G. G., Koptsevich, A. B., \& Sanwal, D. 2001, ApJ, 561, 308

Schild, H. \& Testor, G. 1992, A\&AS, 92, 729

Skrutskie, M. F., Cutri, R. M., Stiening, R., Weinberg, M. D., Schneider, S., Carpenter, J. M., Beichman, C., Capps, R., Chester, T., Elias, J., and 21 coauthors, 2006, AJ, 131, 1163

Slane, P., Chen, Y, Schulz, N., Seward, F. D., Hughes, J. P., \& Gaensler, B. M. 2000, ApJ, 533, L29

Smith, D. A. \& Wang, Q. D. 2004, ApJ, 611, 881

Spitzer, L., Jr. 1982, ApJ, 262, 315

Sun, M., Wang, Z.-R., \& Chen, Y. 1999, ApJ, 511, 274

Tang, S., \& Wang, Q. D. 2005, ApJ, 628, 205

Thielemann, F.-K., Nomoto, K., \& Hashimoto, M.-A. 1996, ApJ, 460, 408

Torii, K., Slane, P. O., Kinugasa, K., Hashimotodani, K., \& Tsunemi, H. 2000, PASJ, 52, 875

Townsley, L. K., Broos, P. S., Feigelson, E. D., Brandl, B. R., Chu, Y.-H., Garmire, G. P., Pavlov, G. G. 2006, AJ, 131, 2140

van der Swaluw, E. 2004, AdSpR, 33, 475

van der Swaluw, E., Achterberg, A., Gallant, Y. A., Downes, T. P., \& Keppens, R. 2003, A\&A, 397, 913

van der Swaluw, E., Downes, T. P., \& Keegan, R. 2004, A\&A, 420, 937

Wang, Q. D. 2004, ApJ, 612, 159

Wang, Q. D., \& Gotthelf, E. V. 1998, ApJ, 494, 623 (WG98)

Wang, Q. D., Gotthelf, E. V., Chu, Y.-H., \& Dickel, J. R. 2001, ApJ, 559, 275

Wang, Q. D., Li, Z.-Y., \& Begelman, M. C. 1993, Nature, 364, 127

Warren, J. S., Hughes, J. P., \& Slane, P. O. 2003, ApJ, 583, 260

Warwick, R. S. et al. 2001, A\&A, 365, L248

Weisskopf, M. C., Hester, J. J., Tennant, A. F., Elsner, R. F., Schulz, N. S., Marshall, H. L., Karovska, M., Nichols, J. S., Swartz, D. A., Kolodziejczak, J. J., \& O’Dell, S. L., 2000, ApJ, 536, L81

Woosley, S. E. \& Weaver, T. A. 1995, ApJS, 101, 181 